This item was submitted to Loughborough's Research Repository by the author.

Items in Figshare are protected by copyright, with all rights reserved, unless otherwise indicated.

\title{
Unsteady predictions of mixing enhancement with steady and pulsed control jets
}

PLEASE CITE THE PUBLISHED VERSION

http://dx.doi.org/10.2514/1.J053374

\section{PUBLISHER}

(c) American Institute of Aeronautics and Astronautics

\section{VERSION}

AM (Accepted Manuscript)

\section{PUBLISHER STATEMENT}

This work is made available according to the conditions of the Creative Commons Attribution-NonCommercialNoDerivatives 4.0 International (CC BY-NC-ND 4.0) licence. Full details of this licence are available at: https://creativecommons.org/licenses/by-nc-nd/4.0/

\section{LICENCE}

CC BY-NC-ND 4.0

\section{REPOSITORY RECORD}

Kamran, Muhammad A., and James J. McGuirk. 2015. "Unsteady Predictions of Mixing Enhancement with Steady and Pulsed Control Jets". figshare. https://hdl.handle.net/2134/18103. 


\title{
URANS Predictions of Mixing Enhancement with Steady and Pulsed Control Jets
}

\author{
Muhammad A. Kamran ${ }^{1}$ and James J. McGuirk ${ }^{2}$ \\ Dept. of Aero. \& Auto. Eng., Loughborough Univ., Loughborough LE11 3TU, UK.
}

\begin{abstract}
URANS CFD predictions are reported for a single round jet at high Reynolds number and high subsonic Mach number $\left(\mathrm{Re}=10^{6}, \mathrm{M}=0.9\right)$ excited by steady and pulsed control jets (CJs). Comparison has been made with experimental validation data to assess the ability of $k-\varepsilon$ based URANS modelling for predicting CJ-driven flow control of near-field jet mixing and potential core length reduction. The well-known overprediction of the clean (unexcited) core length with this level of turbulence closure remains, but, taking this into account, the relative effect of CJs on core length reduction was predicted remarkably well. For example, steady CJs and pulsed CJs in symmetric/antisymmetric modes indicated a core length reduction (relative to the unexcited case) of $43 \% / 45 \% / 60 \%$ in CFD predictions, compared to $48 \% / 40 \% / 51 \%$ in measurements. Comparison of radial profiles showed that URANS modelling was also able to predict the 3D near field behaviour induced by CJs throughout the jet cross-section. The vortex structures produced in the pulsed CJ predictions were compared. Symmetric and antisymmetric modes produced different vortex structures; these caused different levels of enhanced mixing in the two azimuthal modes, and explained the better performance of antisymmetric pulsing.
\end{abstract}

\section{Nomenclature}

$$
\begin{array}{ll}
d_{C J} & =\text { CJ nozzle exit diameter }(\mathrm{m}) \\
D_{n} & =\text { primary jet nozzle exit diameter }(\mathrm{m}) \\
f & =\text { CJ pulsation frequency }(\mathrm{Hz}) \\
S t & =\text { Strouhal number }=f D_{n} / U_{j} \\
S t_{\text {opt }} & =\text { optimum Strouhal number }
\end{array}
$$

\footnotetext{
${ }^{1}$ Research Student, Dept. of Aero. \& Auto. Eng., Loughborough Univ.; currently Assistant Professor, Mechanical Engineering Dept., University of Eng. \& Tech., Peshawar 25030, Pakistan

${ }^{2}$ Prof. of Aerodynamics, Dept. of Aero. \& Auto. Eng., Loughborough Univ., UK.
} 


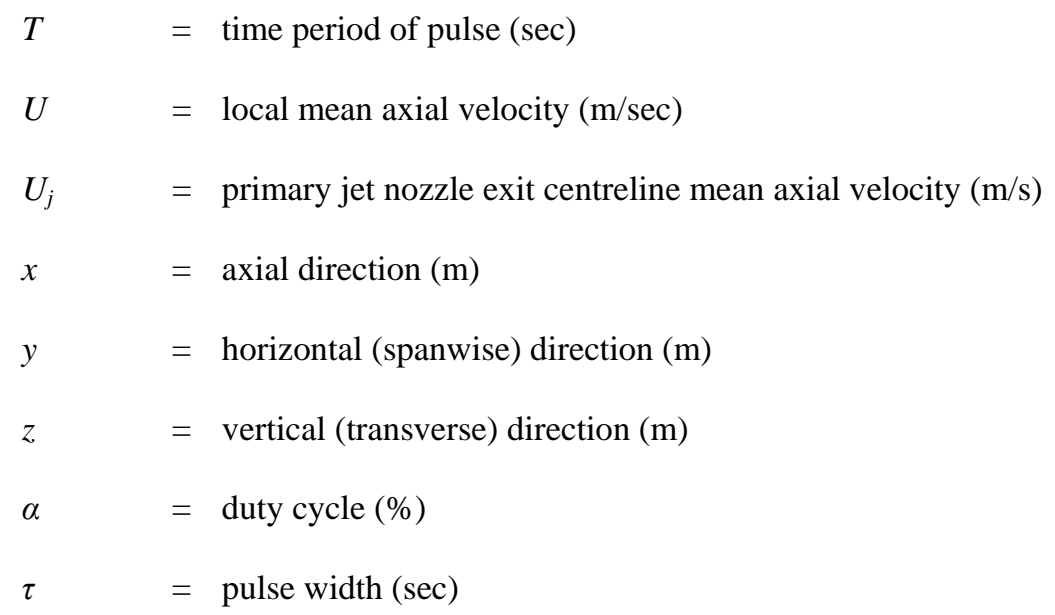

\section{Introduction}

Many engineering design problems involve turbulent jets discharged into and mixing with a surrounding fluid; examples include: (i) gaseous fuel/air mixing in combustion systems, (ii) noise radiation from propulsive jets in civil aviation, and (iii) near-field exhaust plume temperature characteristics in military aerospace applications (to assess infra-red (IR) emissions). The ability to control (i.e. enhance) the jet mixing for performance benefit - lower pollutant formation and increased combustion efficiency, reduced aeroacoustic noise, or reduced IR signature for 'low observable' aircraft - is clearly of significant engineering interest. Control will, however, inevitably involve a trade-off, since techniques and devices that increase mixing will also incur penalties, e.g. thrust loss and added weight, cost, and complexity in propulsion applications; only approaches which offer an 'affordable' solution will be acceptable for introduction into new engine designs. The primary driver of the study reported here is reduced IR signature; this places the focus squarely on enhanced turbulent mixing between a high speed exhaust plume and the surrounding ambient (as opposed to the aeroacoustic (jet noise) application, where understanding of the more subtle (i.e. $4^{\text {th }}$ order space/time correlation) aspects of the turbulent statistics that influence the noise source is required (Afsar et al. [1]). Whilst the temperature field development is obviously of immediate relevance in the context of IR signature, experiments (to be reviewed below) have shown that it is the secondary velocity field induced by the rapid mixing devices introduced into the flow which dominate the scalar field mixing. Thus, in the present work emphasis has been placed in the first instance on the momentum field mixing. Further, the industrial applications listed above imply that high Reynolds number (of order $10^{6}$ based on nozzle exit velocity and diameter) and high Mach number 
(of order 1) characterise the fluid mechanics of the flow, so strong turbulence and compressibility effects are inevitable, and experiments at appropriate conditions are important.

The widespread engineering importance mentioned above means that considerable efforts have gone into research on jet mixing enhancement techniques. Somewhat surprisingly, the majority of this research has been experimental, with only a small number of computational studies appearing in the last decade. Various techniques have been suggested for mixing enhancement, and may be categorised into two groups: passive and active. Passive techniques input no energy into the flow, e.g. exhaust nozzle geometric modifications, from alterations in the jet nozzle exit shape (non-circular cross-section, scarfing, or serrated trailing edge), to the addition of multiple solid tabs or vortex generators penetrating into the main jet flow near nozzle exit. Active techniques inject energy into the flow via a number of discrete control jets (CJs) discharged at an angle to the primary propulsion jet again close to nozzle exit. CJs may be operated under steady flow conditions, or pulsate at a specific pre-defined frequency (open loop control). Acoustic excitation, oscillating membranes, forced momentum jets (steady and pulsed), synthetic jets (zero mass flux unsteady jets), and plasma actuators have all been explored as devices for introducing control jets. Kamran and McGuirk [2] have reviewed this range of previously suggested techniques and the supporting experimental work. Passive techniques (e.g. solid tabs - small protrusions attached to the nozzle wall at the jet nozzle exit to produce counter-rotating streamwise vortex pairs, which encourage ambient fluid to be ingested into the jet core) have been found to introduce considerable drag or thrust loss penalty to achieve sufficient mixing enhancement. This, plus the fact that CJs can be turned off when not required has led to most studies opting for a CJ-based approach. The attraction of the CJ technique was illustrated in Behrouzi and McGuirk [3], who carried out a comparison of tabs and steady CJs (referred to as 'fluidic tabs') - two steady CJs yielded similar mixing enhancement to tabs for a CJ flow rate of just $1 \%$ of the main jet flow rate. The present work is thus focussed on the CJ technique; previous CJ-based experimental work as reviewed in Kamran and McGuirk [2] is summarised next, with additional material on recent CFD studies.

Davis [4] was the first to carry out experiments on the CJ technique for enhanced jet mixing, by placing a pair of steady CJs $180^{\circ}$ apart at nozzle exit. Local velocity reductions up to $30 \%$ for jet Mach number up to 0.7 were reported with CJ mass flow rates as little as $0.5 \%$ of the primary jet. However, the CJ size and momentum in [4] were so large that a significant blockage/primary jet flow reduction was recorded, resulting in a thrust loss that would be unacceptable in any practical application. Behrouzi and McGuirk [3] compared CJ versus solid tab effectiveness initially in incompressible (water tunnel) tests (to allow easy flow visualisation) and subsequently 
using airflow in a high Mach compressible regime. With more practically realisable CJ size and momentum compared to [4], control jet flow penetration slightly greater than the nozzle exit boundary layer thickness was the critical condition to enable strong interaction with the jet shear layer and produce similar jet mixing and spreading rates as two solid tabs. These measurements also clearly indicated that CJs introduced a similar streamwise vortex secondary flow structure as tabs and that this was the primary mechanism for extra mixing, leading to potential core length reduction and increased near field jet centre-line velocity decay rate. The preference for a small number of shear layer excitation sites that had emerged from tab studies (e.g. 2 or at most 4) was confirmed by Behrouzi and McGuirk [3] to apply also to CJs, this being beneficial due to the delay thereby introduced to mutual interaction between the streamwise vortex systems from neighbouring CJs. Lardeau et al. [5] examined the question of enhanced mixing being restricted to the near vicinity of the CJ location by studying the mixing process in both an incompressible plane shear layer and a supersonic/subsonic shear layer perturbed by a single steady transverse CJ with a velocity ratio $V_{\mathrm{cj}} / \mathrm{U}_{\mathrm{j}}=0.89$. The work of [3] and [5] both confirmed that although compressibility effects cannot be completely ignored, CJ characteristics can be captured very well in low Mach/incompressible experiments. Visualisation of the flow in [5] showed that (i) the depth of penetration of the CJ into the supersonic/subsonic mixing layer was highly intermittent, as would be expected from a turbulent CJ flow interacting with a turbulent shear layer, and (ii) the 'near-field' nature of the device effectiveness was related to the rapid axial decay of the streamwise vortex strength. Recently Tamburello and Amitay [6, 7] have used extensive Particle Image Velocimetry (PIV) measurements to explore the detailed interaction between two continuous control jets and an axisymmetric free jet, placing emphasis on the effect of CJ momentum coefficient, and the placing of the CJs relative to main jet nozzle exit. These studies have reinforced the descriptions provided earlier, although only fairly low Reynolds number jets $\left(\sim 10^{4}\right)$ were considered.

The above studies were restricted to steady CJs; pulsed CJs introduce periodic oscillations superimposed on the time-mean continuous flow rate. For maximum effectiveness, a preferred frequency $f$ has been observed in all studies. This may be characterised in non-dimensional terms via an optimum Strouhal number $\left(\mathrm{St}_{\mathrm{opt}}=f \mathrm{D}_{\mathrm{n}} / \mathrm{U}_{\mathrm{j}}\right)$. Surprisingly, a wide range for $\mathrm{St}_{\mathrm{opt}}(0.2-0.6)$ is reported in the literature, but the most commonly observed value is close to 0.3 in most experimental studies. This corresponds to the overall primary jet columnar instability frequency (Crow and Champagne [8]) rather than any mechanism associated with nozzle lip boundary layer/shear layer instability. For pulsed control jets an additional parameter is available for system tuning namely the duty cycle $\alpha$, 
the percentage of time the control jet is 'on' during each pulsation cycle - or alternatively the pulse width (injection time) $\tau$. These parameters are of course related via:

$$
\alpha=\tau / T \quad \text { or } \quad \tau=\alpha / f
$$

where $T$ and $f$ are pulse period and frequency respectively. However, to date for enhanced jet mixing flows, there has been no study of the influence of duty cycle/pulse width on CJ performance.

Raman and Cornelius [9] provided an early investigation of the pulsed technique, using two transverse oscillating jets to excite a $\mathrm{Re}=10^{5}$ rectangular primary jet. For some unstated reason the experiments were performed at the $1^{\text {st }}$ sub-harmonic of the columnar instability mode ( $\mathrm{St} \sim 0.15$ ), with the CJs operating both in phase and out of phase. A 35\% reduction in potential core length and a $60 \%$ increase in the primary jet mass flux at downstream stations in the jet near field were achieved compared to an unexcited ('clean') jet. It is likely that this study was more aimed at using the pulsed CJs to impinge on the primary jet and force global primary jet flapping as opposed to using unsteady CJs to increase streamwise vorticity enhanced mixing. Parekh et al. [10] were perhaps the first to apply the pulsed CJ technique to excite unsteady streamwise vorticity by oscillating two slot jets in axisymmetric mode (in phase, sometimes referred to as the 'varicose' mode) and anti-symmetric mode $\left(180^{\circ}\right.$ out of phase, sometimes described as the' sinuous' mode). Peak CJ velocities $\sim 30 \%$ of the primary jet velocity ( $2 \%$ of primary mass flow) were employed to excite the primary jet, again at the $1^{\text {st }}$ sub-harmonic. A large increase in spreading rate of the primary jet was achieved; the data showed that pulsed CJs were extremely effective in reducing the potential core length and were more effective in anti-symmetric compared to symmetric excitation mode. The work of Ibrahim et al. [11] used a primary jet at $\mathrm{Re}=1.5 \times 10^{5}$ and also showed that anti-symmetric injection was preferable in terms of centreline velocity decay. However, ignoring the earlier knowledge gained from multiple tab and CJ flow studies on optimum vortex source numbers, the experiments of [11] used 12 control jets around the nozzle circumference to excite the jet flow, so it is unlikely that this study revealed anything about optimum operational conditions. Behrouzi, McGuirk and Feng [12, 13], placing emphasis on practically realisable aspects of the excitation system, adopted just two CJs and limited the CJ mass flow to a maximum of $1 \%$ of primary flow, adjusting the CJ nozzle diameter to obtain a velocity ratio $V_{c j} / U_{j} \sim 1$ in order to optimise CJ penetration and enable maximum interaction between CJ flow and primary jet shear layer. The data obtained showed that mixing effectiveness with a pair of pulsed CJs $180^{\circ}$ out of phase was as good as achieved with a pair of solid tabs for a total CJ flow of only $0.5 \%$ of the primary jet (halving the mass flow required by steady CJs for the same effect). The performance of the pulsed CJs was strongly influenced by the CJ flow rate, pulse frequency and phase, and these 
parameters had to be carefully chosen for optimal performance (i.e., maximum increased mixing for minimum CJ mass flow). Kamran [14] and Kamran and McGuirk [2], in their study of steady and pulsed CJ excitation effects on mixing of a Mach 0.9 jet argued that the advantage of pulsed CJs over steady CJs could only be properly assessed and compared if the peak amplitude of pulsed CJ velocity did not exceed the steady CJ velocity amplitude. This was not followed in any of the studies reported above, in which the mass flow rates of both steady and pulsed CJs were the same, resulting in higher velocity amplitude for pulsed CJs compared to steady CJs. Kamran and McGuirk [2] reported a potential core length reduction of $40 \%$ with steady CJs, increasing to $50 \%$ with pulsation; similarly, the jet cross-sectional area at the end of the near-field $\left(x / D_{n}=10\right)$ increased by $25 \%$ (steady) and $30 \%$ (pulsed). The introduction of pulsation halved the required CJ mass flow rate needed to achieve the same (in fact slightly increased) mixing enhancement compared to steady CJs. Finally, two papers which used synthetic rather than forced momentum jets may be mentioned as still relevant to the current study. Firstly Ritchie et al. [15], motivated by an application related to active control of gaseous fuel-air mixing, conducted both PIV measurements of the velocity field and Planar Laser Induced Fluorescence (PLIF) measurements of the scalar mixing field. A comparison of velocity and scalar data showed clearly that the large-scale flow structures visible in the scalar data correlated well with the velocity flow features. Secondly, Tamburello and Amitay [16] extended their PIV studies to pulsed (synthetic) jet excitation, again at low Re (only 6600), but confirming (and quantifying) the presence of strong streamwise vorticity as the crucial mixing enhancement mechanism, and identifying an optimum Strouhal number $\mathrm{St}_{\mathrm{opt}}=0.32$ for low pulsed jet momentum coefficient, and a saturation mechanism for higher pulsed jet momentum.

As already noted above there has been rather little application of CFD to analyse the effects of either steady or pulsed CJs on near field jet aerodynamics. Even more surprisingly, first attempts to apply CFD modelling all adopted Direct Numerical Simulation (DNS), rather than the conventional RANS (Reynolds Averaged NavierStokes) technique. This of course severely limited the Reynolds numbers that could be used. Freund and Moin [17] computationally forced a Mach 0.9 but low Reynolds number laminar jet $(\mathrm{Re}=3600)$ with two steady slot jets that extended $90^{\circ}$ around the jet circumference at Strouhal numbers of 0.2 and 0.4. The simulations showed that, similar to solid tabs, the CJs make the jet highly non-axisymmetric, causing a rapid spread perpendicular to the CJ axis and a suppression of spread along the CJ axis. The low Re and non-typical CJ and main jet conditions make this study of little quantitative relevance to the present application. Hilgers and Boersma [18] also adopted a DNS approach but excited the main jet via global inlet forcing rather than at discrete azimuthal locations. A combination of axial and helical forcing was introduced, as well as an optimisation algorithm to identify the best combination of axial and 
helical modes; the axial (varicose) mode was found to be dominant. The main jet Reynolds number of the study of Lardeau et al. [19] was again dictated by the DNS approach - only 1500 - but this was the first work where two discrete control jets were used, although these were angled at $45^{0}$ to the main jet, had diameters as large as $1 / 4$ of the main jet and had a combined mass flow as high as $10 \%$ of the main jet. For the first time in CFD both continuous and pulsed jets were considered, with the pulsing frequency chosen to match $S t=0.2$, and both in phase and out of phase modes simulated. The scenario modelled was certainly much closer to the regime of interest here than the DNS CFD studies of [17] and [18], but many of the operating parameters were far removed from the design range of interest. This study did display a strong counter-rotating vortex pair fundamental to enhanced mixing.

Smith et al. [20] were the first to conduct a URANS (Unsteady RANS) CFD study of the effect of pulsed blowing on mixing enhancement of a hot jet for a subscale and axisymmetric turbojet engine nozzle. Two pulsed jets were used, but again the rather untypical size of a $90^{\circ}$ circumferential arc was chosen. The pulsed jets were excited in the anti-symmetric (out of phase) mode with a total mass flow of $3 \%$ of the primary jet. No explanation of how the pulse frequency was selected was provided. A large scale flapping mode of the main jet was observed with amplitude well in excess of one nozzle diameter. Although significant reduction in potential core length was reported, no comparison with measurements was provided, and the chosen operating parameters of the CJs seem so far from those of relevance to the current interest (high mixing enhancement for small CJ 'expenditure') as to be effectively irrelevant.

Behrouzi and McGuirk [3] studied the effect of two diametrically opposed steady CJs on an incompressible round jet using a k- $\varepsilon$ RANS approach to explore the mechanism of CJ effectiveness, and compared their findings against similar predictions for two tabs. Fig. 1 shows predicted axial velocity contours and secondary flow vectors taken from [3] at a location $3 \mathrm{D}_{\mathrm{n}}$ downstream of nozzle exit. The symmetry of $\mathrm{CJ}$ and tab placement (at 12 and 6 o'clock positions) meant that just a quadrant of the nozzle flow could be considered. These predictions underline the similarity of the mechanisms involved in tabs and steady CJ flow perturbation, viz. injection into the main jet shear layer of a counter-rotating pair of streamwise vortices (for each tab/CJ), causing inward movement of ambient flow along the line joining CJs and ejection of jet flow outward in the orthogonal direction. The originally circular crosssectional shape is distorted by the streamwise vortex field as is clearly visible in Fig.1; the increase in jet/ambient interfacial area this brings about is one of the main contributions to enhanced mixing. There is also close similarity between the secondary flow fields created by tabs and control jets. Unfortunately [3] did not provide experimental validation of the predicted CJ flow patterns, so their quantitative accuracy is uncertain. 

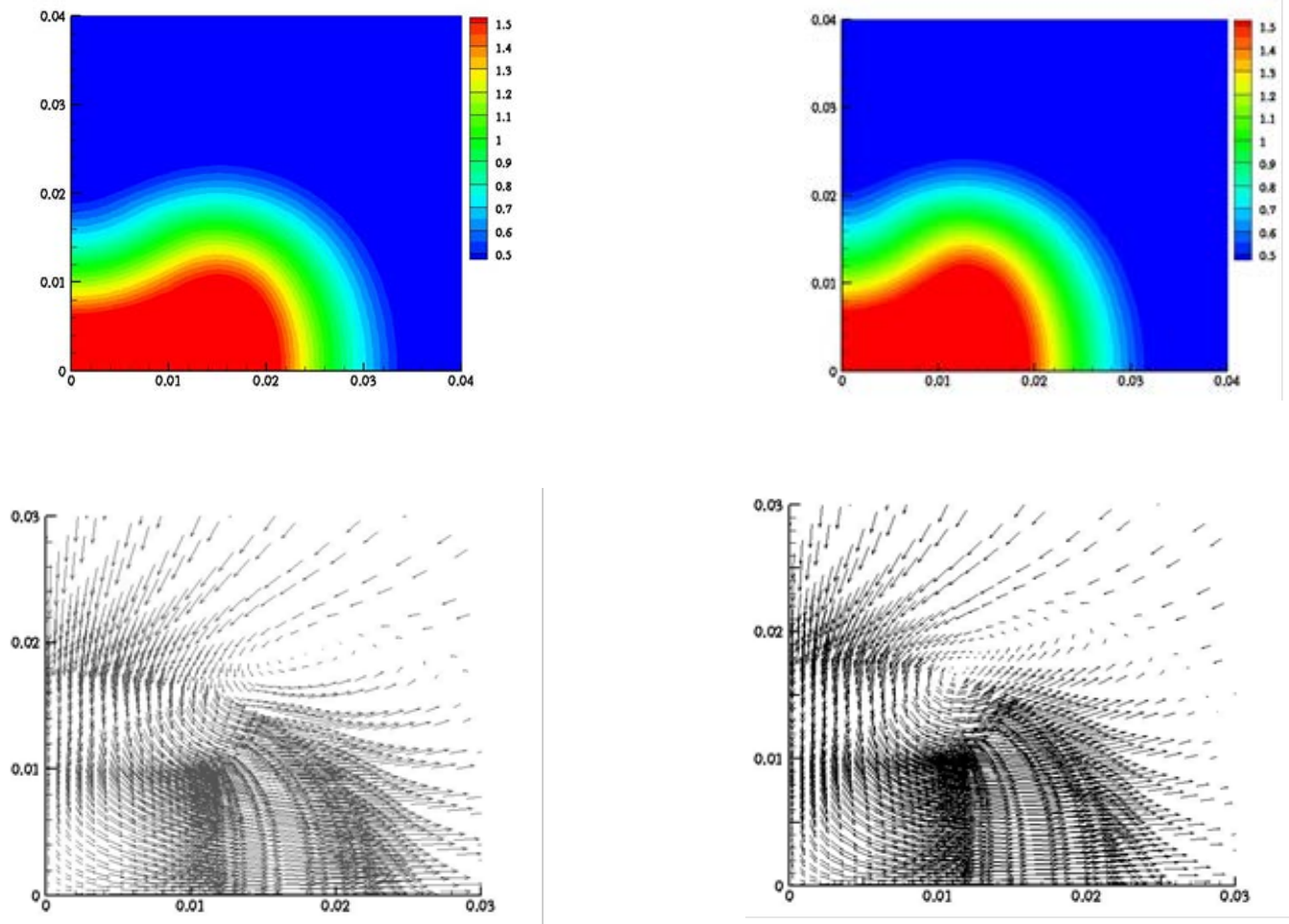

Fig. 1 Predicted [3] velocity contours/secondary flow vectors; $x / \mathbf{D}_{n}=3.0$, solid tab (left), fluid tab (right).

The most recent CFD studies relevant to CJ-enhanced mixing are by Chauvet et al. [21], [22], where combined experimental and numerical investigations were conducted. Only steady CJs were considered, and the geometry chosen was significantly more complex than in all other works reviewed above, much closer to a realistic engine installation. The primary jet issued from a convergent nozzle operating at either moderate (3.1) or strong (5.0) Nozzle Pressure Ratios (the baseline jet was an under-expanded flow containing embedded compression/expansion waves, and a Mach disc at the higher NPR). This was surrounded firstly by an annular subsonic stream to represent the propulsion nozzle ventilation flow, and secondly by a co-axial wind tunnel flow at Mach 0.8 to represent a flight stream. Prior work described above emphasising the importance of a small number of non-interfering secondary flow injectors was not followed since up to 8 CJs were included. Unsurprisingly, the results from both experiments and CFD indicated that 2 and 4 injectors produced the best enhanced mixing performance, with the thrust loss of the 2-injector case being $60 \%$ of the 4 -injector results. 
In terms of CFD modelling [21] adopted a RANS approach, using a 1-equation Spalart-Allmaras turbulence model, and was carried out for complete $360^{\circ}$ nozzle geometry, leading to an 8 million node mesh. The predictions were only compared with measurements along the jet centreline. Whilst enhanced mixing was predicted, discrepancies between computations and experiments were present. In particular it was noted that the potential core length was overpredicted in both the baseline jet and the jet with steady CJs. This is a well-known flaw in eddy viscosity-based RANS turbulence models when applied to the analysis of propulsive jet near-field mixing. This topic has been extensively reviewed by Georgiadis et al. [23], and Georgiadis and DeBonis [24]. Their main conclusions were: (i) standard two-equation models (with model constants calibrated against a wide range of 2D shear flows to give optimum overall accuracy) consistently overpredicted round jet potential core length due to inhibited initial turbulence growth rate and produced a too rapid initial centreline decay rate relative to experimental data, (ii) several attempts to introduce modifications and corrections to improve accuracy for near-field jet mixing were shown to have rather variable results. For example, the modified k- $\varepsilon$ model of Thies and Tam [25] was successful in improving core length prediction accuracy, but downstream of the potential core the decay rate of axial velocity was now significantly too slow in comparison with measurements; further, all model modifications studied showed inadequacy in their ability to improve the accuracy of prediction of the development of turbulence energy. The final conclusion, therefore, was that a satisfactory RANS model capability for mean and turbulent near field flowfields in propulsion jet flow remains elusive. This led Chauvet et al. [22] to attempt to improve predictive accuracy by adopting a zonal hybrid RANS/Large Eddy Simulation (LES) CFD approach (Detached Eddy Simulation (DES)). This did improve the potential core prediction, but only after the length scale in the LES subgrid-scale model had been adjusted accordingly; before this the RANS and DES results were rather similar. With the adjusted model, the radial profiles with and without CJ forcing agreed well with measurements, and produced a quantitatively accurate picture of mixing rates. It was pointed out, however, that the cost of DES was 20 times that of the RANS solution.

Based on this summary of previous CFD studies, the work reported here was aimed at extending the work on computational analysis of CJ-enhanced mixing. There is a clear shortage of CFD analysis - in particular there have been no detailed studies of the effectiveness of the URANS CFD approach for pulsed CJ flow. The high computational expense illustrated in [22] of the more advanced approaches to turbulence modelling means that there is still significant interest in use of URANS CFD for design purposes. The problem of potential core length overprediction with RANS modelling remains an issue. First attempts to apply the LES approach have shown 
(Andersson et al [25]) that this aspect is not easily cured - in fact LES was observed to underpredict (by 15\% [25]) the potential core length. Careful treatment of the strongly accelerating flow inside the nozzle to ensure correct turbulence conditions in the thin boundary layer at nozzle exit can produce correct core length prediction - see Wang and McGuirk [26] for a non-excited rectangular jet. Thus, although both DES and LES have shown promise at resolving the potential core prediction problem, the large computational cost of these techniques means it is difficult to see these being used currently in a typical industrial design cycle. It is of interest therefore to examine the question of whether a URANS method is capable of quantitatively assessing the performance enhancement brought about by pulsed CJs. The potential core problem will remain, but if URANS can predict accurately the relative effect of pulsed CJs compared to the baseline case, then a useful design role could is possible. The rest of the paper describes the application and validation of URANS for the Mach 0.9 cold jets studied experimentally by Kamran and McGuirk [2] and Kamran [14] for baseline (unexcited), steady, and pulsed CJ excitation. Given that no firm recommendation on k- $\varepsilon$ model improvements was made in [23], [24], the standard model was employed in the predictions shown below.

\section{Computational Techniques and Procedures}

\section{Mathematical Model}

The Favre-averaged density-weighted technique (indicated by a tilde ( $)$ ) as commonly used for modelling compressible turbulent flows is adopted in the present work to separate turbulent fluctuations (") from the nonturbulent flow. In a URANS formulation, a triple decomposition is adopted; thus for any instantaneous variable $\varphi$ :

$$
\varphi\left(x_{i}, t\right)=\tilde{\varphi}\left(x_{i}, t\right)+\varphi^{\prime \prime}\left(x_{i}, t\right)=\varphi^{M}\left(x_{i}\right)+\varphi^{P}\left(x_{i}, t\right)+\varphi^{\prime \prime}\left(x_{i}, t\right)
$$

In URANS the first term on the right hand side is still a function of time, but it is assumed that the time-variation is not stochastic as in the second term, but is only due to the coherent (single frequency) periodic variations caused by the time-varying boundary conditions introduced by the pulsed control jet. This first term may be further split into $\varphi^{M}$ representing the long time -mean and $\varphi^{P}$ representing the periodic component; it is assumed that these two components are uncorrelated in time with the turbulent fluctuation. For a clean jet or steady control jets $\varphi^{P}$ is absent. The governing flow equations become (an overbar (־) indicates an un-weighted time- averaged quantity):

$$
\frac{\partial \bar{\rho}}{\partial t}+\frac{\partial\left(\bar{\rho} \tilde{u}_{i}\right)}{\partial x_{i}}=0
$$




$$
\frac{\partial\left(\bar{\rho} \tilde{u}_{i}\right)}{\partial t}+\frac{\partial\left(\bar{\rho} \tilde{u}_{i} \tilde{u}_{j}\right)}{\partial x_{i}}=-\frac{\partial \bar{p}}{\partial x_{i}}-\frac{\partial\left(\bar{\rho} \widetilde{u_{i}^{\prime \prime} u_{j}^{\prime \prime}}\right)}{\partial x_{i}}+\frac{\partial \bar{\tau}_{i j}}{\partial x_{i}}
$$

$\bar{\tau}_{i j}$ is the time-mean viscous stress, with $\mu$ the molecular viscosity and $\overline{S_{i j}}$ is the mean strain rate :

$$
\bar{\tau}_{i j}=2 \mu \bar{S}_{i j}-\frac{2}{3} \mu \delta_{i j} \frac{\partial \bar{u}_{k}}{\partial x_{k}} \quad \bar{S}_{i j}=\frac{1}{2}\left(\frac{\partial \bar{u}_{i}}{\partial x_{j}}+\frac{\partial \bar{u}_{j}}{\partial x_{i}}\right)
$$

these terms are usually negligible at high Re. Note that in the unheated jet experiments of Kamran \& McGuirk [2] the total temperature of primary jet, CJ flow and the surrounding stagnant ambient fluid were the same. Thus, in the current predictions a simplification has been made that avoids the need to solve an energy equation in addition to eqns. (2) and (3). Under the conditions of the experiment, it may be assumed that the average total temperature is everywhere constant. This allowed an assumption of constant total enthalpy to be adopted, and using the equation of state for an ideal gas the mean density could be calculated from the mean velocity and static pressure fields (ignoring the fluctuating kinetic energy contribution):

$$
\tilde{H}=\frac{\gamma}{(\gamma-1)} \frac{\bar{p}}{\bar{\rho}}+\frac{1}{2} \tilde{u}_{i} \tilde{u}_{i}=\text { constant }
$$

This is an approximation when the flow is unsteady, but proved acceptable when comparing with both mean and turbulence measurements for a Mach 0.9 jet as reported in Afsar et al. [1].

The turbulent Reynolds stresses in eqn. (3) are calculated using a high Re k- $\varepsilon$ model:

$$
\begin{gathered}
-\bar{\rho} \widetilde{u_{i}^{\prime \prime} u_{j}^{\prime \prime}}=\mu_{t}\left(\frac{\partial \tilde{u}_{i}}{\partial x_{j}}+\frac{\partial \tilde{u}_{j}}{\partial x_{i}}\right)-\frac{2}{3} \delta_{i j}\left(\mu_{t} \frac{\partial \tilde{u}_{i}}{\partial x_{i}}+\bar{\rho} \tilde{k}\right) \\
\frac{\partial(\bar{\rho} \tilde{k})}{\partial t}+\frac{\partial\left(\bar{\rho} \tilde{u}_{i} \tilde{k}\right)}{\partial x_{i}}=-\frac{\partial}{\partial x_{i}}\left(\left(\frac{\mu_{t}}{\sigma_{k}}\right) \frac{\partial \tilde{k}}{\partial x_{i}}\right)+P_{k}-\bar{\rho} \tilde{\varepsilon} \\
\frac{\partial(\bar{\rho} \tilde{\varepsilon})}{\partial t}+\frac{\partial\left(\bar{\rho}_{i} \tilde{\varepsilon}\right)}{\partial x_{i}}=-\frac{\partial}{\partial x_{i}}\left(\left(\frac{\mu_{t}}{\sigma_{\varepsilon}}\right) \frac{\partial \tilde{\varepsilon}}{\partial x_{i}}\right)+\frac{\tilde{\varepsilon}}{\tilde{k}}\left(C_{\varepsilon 1} P_{k}-C_{\varepsilon 2} \bar{\rho} \tilde{\varepsilon}\right) \\
\mu_{t}=\bar{\rho} C_{\mu} \frac{\tilde{k}^{2}}{\tilde{\varepsilon}} \quad P_{k}=\overline{\rho u_{i}^{\prime \prime} u_{j}^{\prime \prime}}\left(\frac{\partial \tilde{u}_{i}}{\partial x_{j}}\right)
\end{gathered}
$$

The choice of this model was influenced by the fact that the majority of the turbulence activity that needs to be captured accurately in the present application occurs in free-shear regions, and this model is generally accepted as 
optimum for such flows from available two-equation closures. For the near-wall flow inside the nozzle high Re wall functions have been used. Finally, all model constants have been given conventional values as suggested by Launder and Spalding [28]:

\begin{tabular}{|c|c|c|c|c|}
\hline $\mathbf{C}_{\boldsymbol{\mu}}$ & $\mathbf{C}_{\varepsilon 1}$, & $\mathbf{C}_{\varepsilon 2}$, & $\boldsymbol{\sigma}_{\mathbf{k}}$ & $\boldsymbol{\sigma}_{\varepsilon}$ \\
\hline 0.09 & 1.44 & 1.92 & 1.0 & 1.30 \\
\hline
\end{tabular}

\section{Solution Domain, Mesh and Boundary Conditions}

The physical problem to be studied consists of a single round subsonic jet discharging into a stagnant ambient at NPR $=1.7$, with two added diametrically opposed Control Jets (CJs) injecting orthogonal to the primary jet axis at a CJ supply pressure of 2 bar and with a pulsation frequency of $1050 \mathrm{~Hz}(\mathrm{St}=0.22)$ at a duty cycle of 0.32 . URANS numerical predictions for the 'clean' (no control jet flow) primary jet flowfield, with steady CJs or excited using pulsed CJs were carried out using the in-house code DELTA. This is a multi-block 3D structured (non-orthogonal) mesh code based on a co-located finite-volume implicit pressure-correction algorithm. A 2nd-order accurate discretisation of the convective fluxes in the momentum, $\mathrm{k}$, and $\varepsilon$ equations was selected using a limited form of the QUICK differencing scheme. The transient equations were solved even when the boundary conditions allowed a statistically steady solution; the time-step was determined such that only a small number (typically 5) of inner iterations were required to converge the Poisson equation used to satisfy continuity; this corresponded to a time-step of $1.0 \times 10^{-6}$ secs. This was halved for pulsed CJ cases, leading to the fine temporal resolution of almost 2000 time steps per pulse for the frequency investigated. The pulsed calculations were run (starting from the clean CJ solution as an initial condition) until the start-up disturbance had passed through the entire solution domain (at least 2 flowthrough times). A long time-averaged mean of the solution was then calculated, averaging over 8 pulsation cycles. Full details of the code can be found in Page et al. [29]. Evidence of the code's ability to predict a wide range of relevant flow conditions is available for incompressible jets [12] high Mach subsonic jets [1] and under-expanded jets [30].

Symmetry conditions were used to reduce the computational overhead and $a 180^{\circ}$ solution domain sector was chosen for the current study. The clean jet, steady CJ, or in phase pulsed CJ cases could have been predicted using a $90^{0}$ sector (as in Fig. 1) but the anti-symmetrical (out of phase) pulsating case required the larger sector and for 
convenience all calculations used the same solution domain. The nozzle geometry used in [2] was a simple convergent conical design with an exit diameter $D_{n}=60 \mathrm{~mm}$ and a nozzle internal half angle of $11^{\circ}$; a short (30mm) parallel extension was included at nozzle exit to remove the presence of a vena contracta [14]. The mesh consisted of $\sim 1$ million cells distributed over 24 blocks, with the computational domain extending $\sim 2 D_{n}$ upstream from nozzle exit inside the nozzle, $15 D_{n}$ in the axial direction downstream of nozzle exit, and $4 D_{n}$ in the two cross-stream directions. The co-ordinate system used to describe the geometry and present all results has $\mathrm{x}$ as axial direction, $\mathrm{z}$ as vertical, and y as horizontal cross-stream directions. Fig. 2 illustrates the domain size and block structure (CJs not yet included in this figure). The grid resolution in the cross-stream directions and the H-grid/O-grid topology used for the primary jet core to avoid any jet centreline singularity and stiffness problems are shown in Fig. 3. All calculations were carried out with the primary jet discharging into a stagnant ambient as in the experiments. For convenience of imposing boundary conditions for the flow entrained by the jet from upstream, an extra 'entrainment flow' block was included around the nozzle just upstream of the nozzle and close to nozzle exit, details given below.

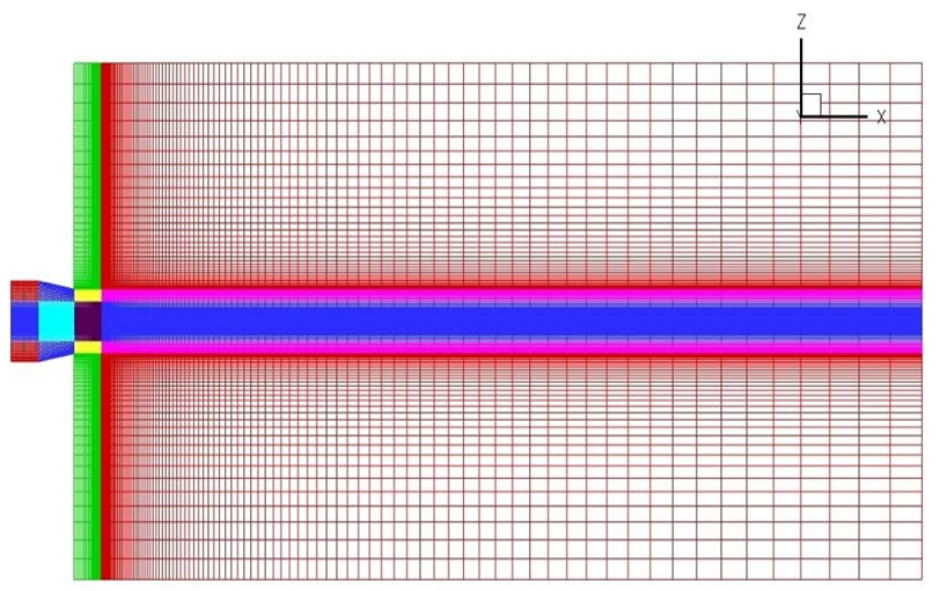

Fig.2 Solution domain and mesh structure $-x-z$ plane

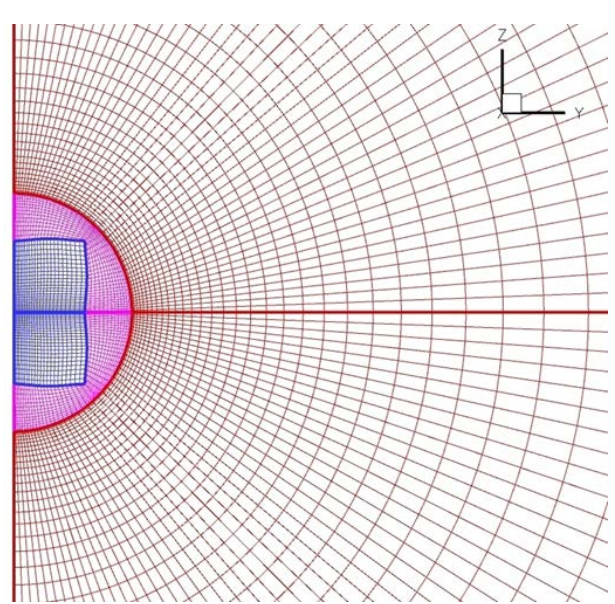

Fig. 3 Mesh structure $-\mathrm{y}$-z plane

To capture the two CJ inflows, it was necessary to create two extra blocks attached appropriately at nozzle exit, see Fig.4. These were 'virtual' blocks - as indicated in Fig. 4 the CJ blocks were physically connected to the internal nozzle grid block at the nozzle wall interface. However, although the CJ blocks protruded radially outward and were geometrically 'inside' the external entrainment flow block attached around the nozzle as described above, no 
connectivity information was supplied between these blocks, so the flow in each was unconnected. The CJ blocks consisted of 10 planes of cells extending radially out for a distance of $6 d_{C J}\left(d_{C J}=2 \mathrm{~mm}\right.$ in the experiment); the CJ inlet condition was fixed on the radially outermost plane in the CJ blocks.

In terms of numerical resolution adequacy, it should be noted that only the near field (axial distance $<15 D_{n}$ and cross-stream distance $<4 D_{n}$ ) is being considered, considerably less than in most jet flowfield studies; preliminary calculations with $50 \%$ less grid nodes ( 0.5 million cells) showed little influence of the mesh (see Kamran [14]). Test calculations with an increasing number of cells over the (whole) CJ cross-section (from 6 to 24), little difference was seen beyond 12 cells. For temporal resolution, as noted above the computational $\Delta t$ for pulsed cases corresponded to 2000 timesteps per pulse; again halving this number did not affect the results [14].

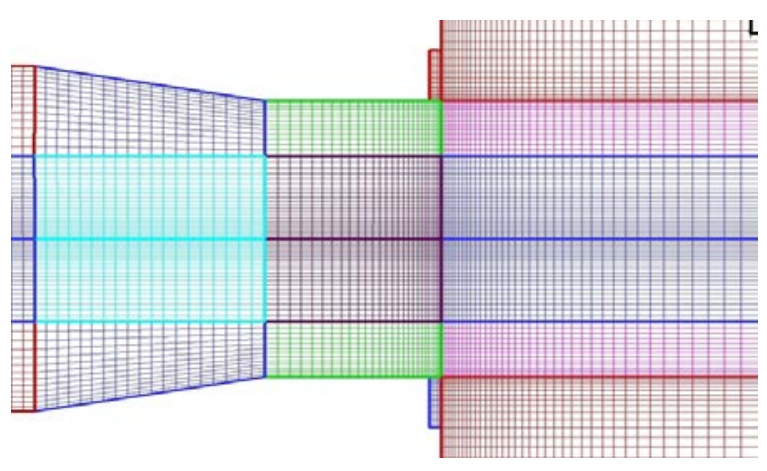

Fig. 4 Mesh zoom-in near nozzle exit showing two

\section{CJ blocks at 12 and 6 o-clock}

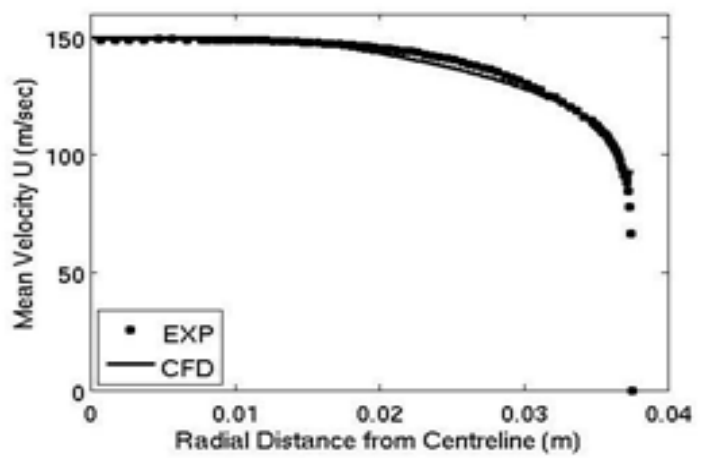

Fig. 5 Primary jet nozzle inlet axial velocity from precursor calculation

The inlet boundary conditions for the primary nozzle were arranged to correspond to those measured in [2] at an NPR of $\sim 1.70$ to achieve a primary jet Mach number of 0.9. Nozzle inlet distributions of axial velocity, turbulence energy and dissipation rate were determined by performing a precursor simulation for a circular pipe with diameter equal to the nozzle inlet size $(75 \mathrm{~mm})$, extracting the solution which matched best the measured axial velocity at nozzle inlet in the experiments, see Fig.5; further details can be found in [14]. Fixed (uniform) total pressure (at a level of 2 bar), as well as uniform $\mathrm{k}$ and $\varepsilon$ (low values, corresponding to a turbulence intensity of $\sim 0.5 \%$ and a turbulent viscosity equal to the molecular viscosity (enabling $\varepsilon$ to be calculated from $\mathrm{k}$ and $\mu_{\mathrm{t}}$ ) were specified at both CJ inlets for steady CJs, allowing the CJ flow to expand from this supply state to whatever static pressure level was predicted to occur at the nozzle exit. For pulsed CJs, a time-varying total pressure condition was applied again taken from measurements [14]. Fig. 6 shows the measured pressure with a duty cycle of 32\%; the fitted curve specified the 
time-varying CJ inlet total pressure. The pulsation frequency (Strouhal number $S t$ ) was fixed at $1050 \mathrm{~Hz}(S t=0.22)$ for the calculations presented below; a single frequency was sufficient - stability was within 1\% as shown by Fig. 7 (for a frequency of $1500 \mathrm{~Hz}$ ). Whether the CJs pulsed in phase or out of phase was controlled explicitly via the CJ boundary conditions.

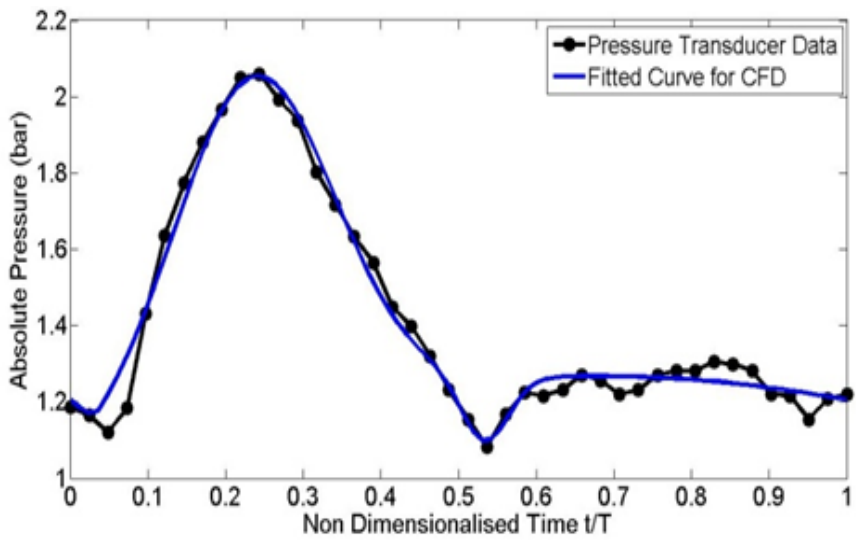

Fig. 6. Inlet condition pulsed CJs - duty cycle $\alpha=32 \%$

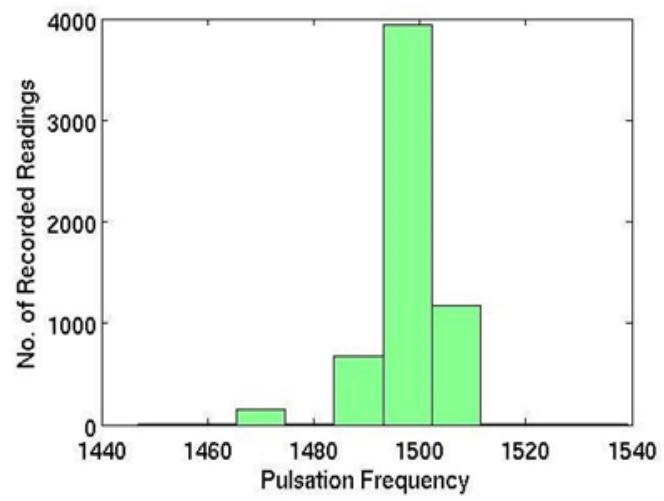

Fig. 7. Pulsed CJs - frequency $(1500 \mathrm{~Hz})$

At the entrainment flow boundary a small axial velocity (10\% of the primary jet velocity) was prescribed to avoid flow recirculation on this boundary. The other boundary conditions were fixed at ambient static pressure and a symmetry plane condition.

\section{Results}

Numerical predictions have been performed for: (i) the clean Mach 0.9 jet (Reynolds number based on $D_{n}$ and the measured nozzle exit peak velocity of $1.33 \times 10^{6}$ ), followed by an examination of the changes brought about in the primary jet flowfield due to introduction of: (ii) steady CJs at a feed pressure of 2 bar, and (iii) pulsed CJs at the same feed pressure but oscillating with the duty cycle of 32\% shown in Fig. 6 at a Strouhal number of 0.22 both in phase and out of phase. In each case the results are analysed via detailed comparison of the equivalent cases studied experimentally by Kamran and McGuirk [2] and Kamran [14] (Note: experimental accuracy quoted in [14]: +/-2\%).

\section{i. Clean Jet - No Control}

To characterise the baseline jet mixing behaviour, Fig. 8 presents predicted mean axial velocity contours for the axisymmetric jet on the $\mathrm{x}-\mathrm{z}$ plane $(\mathrm{y}=0)$. The growth of the shear layer on the jet edge and the length of the potential 
core region are clearly illustrated in this contour plot. To assess the ability of the k- $\varepsilon$ turbulence model to predict the jet near field development, a comparison of the predicted centreline axial velocity with the experimental data is given in Fig. 9a. The predicted velocity magnitude at the nozzle exit plane centreline $\left(\mathrm{U}_{\mathrm{j}}\right)$ matches well with the measured value; this is evidence that the care taken, using the precursor calculation described above, to provide an experimentally matched velocity profile at nozzle inlet has worked well. As expected using an eddy viscosity model, the potential core is predicted to be too long $-8.2 \mathrm{D}_{\mathrm{n}}$ (CFD) compared to $6.2 \mathrm{D}_{\mathrm{n}}$ (Expts.), an error of $32 \%$. The potential core length is here defined as the axial location on the nozzle centreline where the velocity falls below 99\% of $\mathrm{U}_{\mathrm{j}}$. This confirms that with the standard high Re model constants, the annular shear layer around the jet periphery is predicted to grow too slowly. The velocity decay rate downstream of the potential core is initially predicted to be substantially steeper than measured - caused by the sudden increase in predicted turbulence at potential core end, see Fig.9b; after this it is reasonably well predicted in comparison with measured data (the tail-off in the predicted curve beyond $\mathrm{x} / D_{n}=11$ is an upstream 'contaminating' effect of the outlet boundary condition imposed.

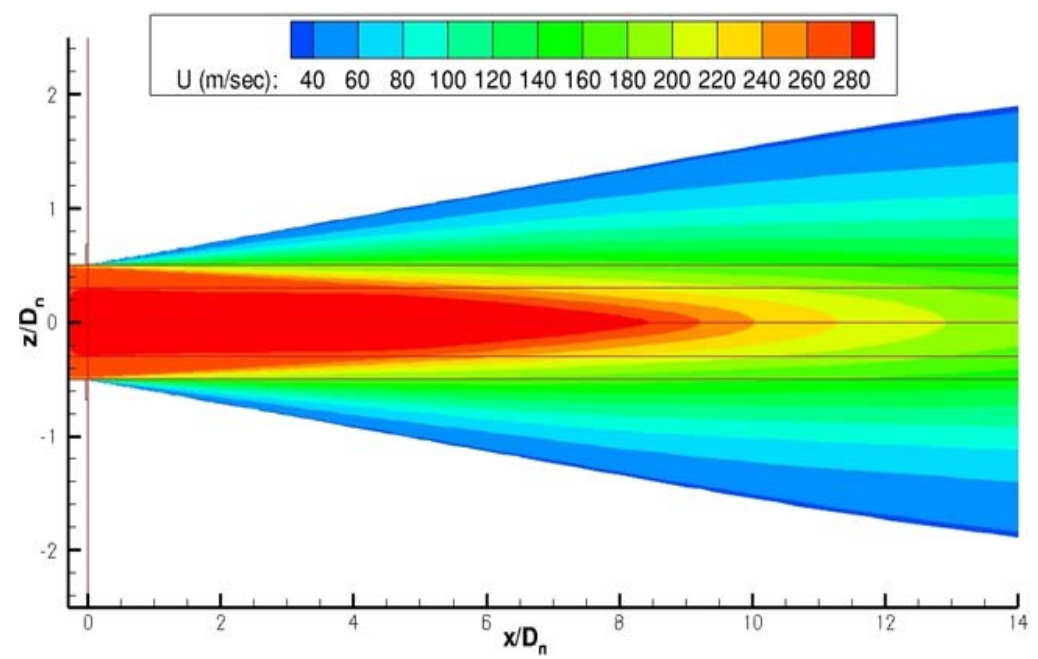

Fig. 8 Mean axial velocity contours 


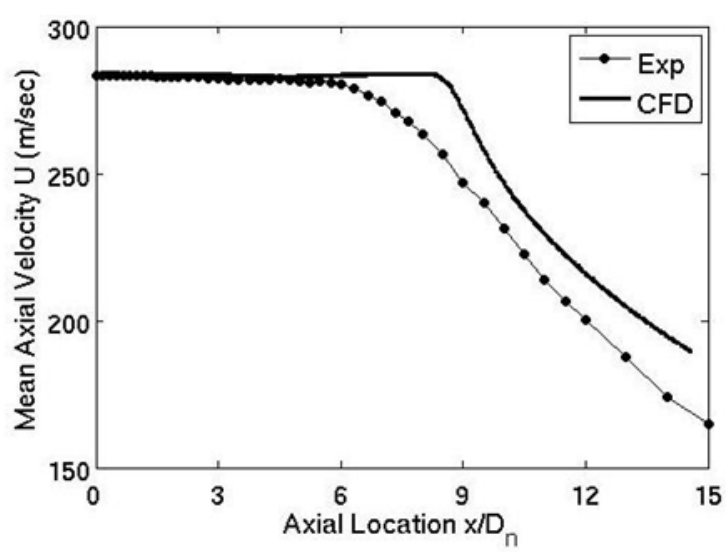

Fig. 9a Mean axial velocity on jet centreline CFD and Expts. [2]
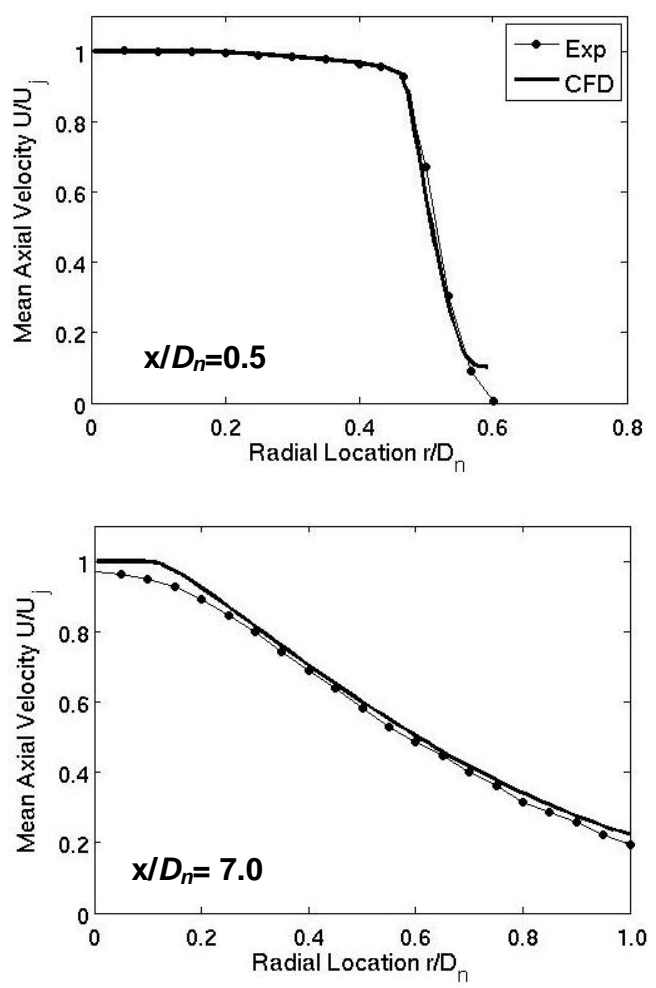

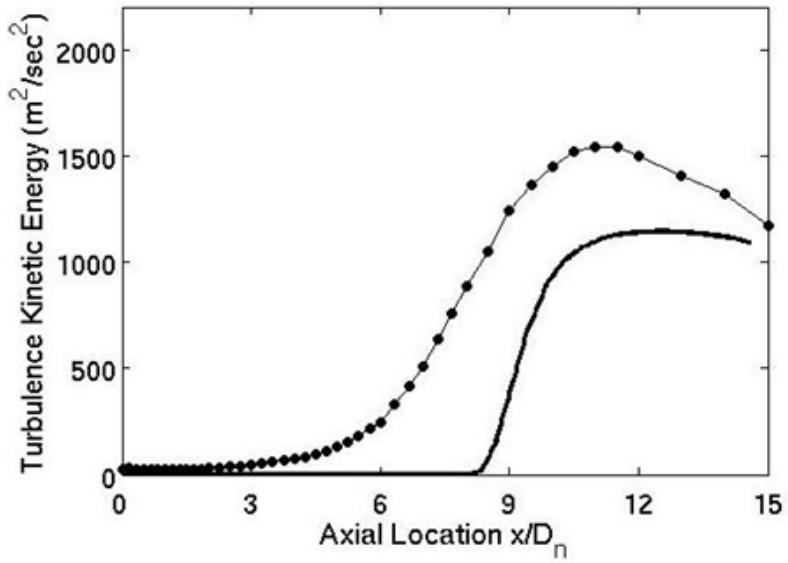

Fig. 9b Turbulence energy on jet centreline CFD and Expts. [2]
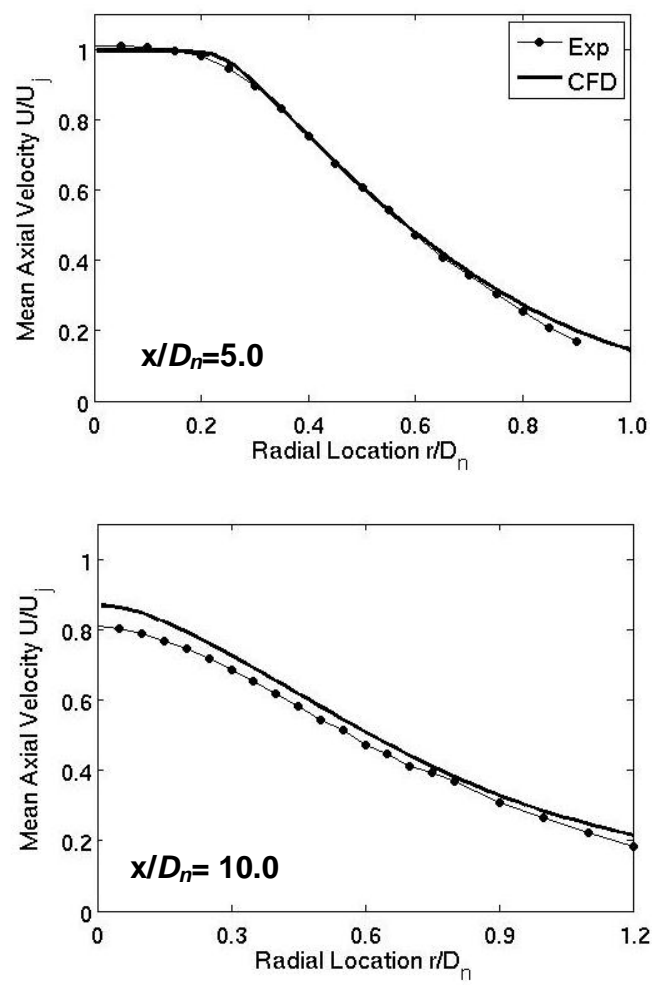

Fig.10 Non-dimensional mean axial velocity profiles for clean jet- CFD and Expts. [2]

The potential core length discrepancy is certainly a problem, but examination of the change in shape of the radial velocity profiles due to near field momentum mixing shows the turbulence model is not performing too badly, see Fig. 10. The development of the mean velocity profile is reproduced well up to $\mathrm{x} / D_{n} \sim 7$; in particular the location of 
the outer jet edge is well captured. Downstream of this the peak velocity magnitude is higher than the experimental data. At $\mathrm{x} / D_{n}=10$ the profile comparison indicates that the velocity decay is slightly slower in the predictions, i.e. the jet/ambient mixing is underpredicted even beyond the potential core end.

\section{ii. Steady Control Jets}

The injection of two steady CJs into the primary jet affects the development of the shear layer and hence the jet/ambient mixing, with jet contraction observed along the line joining CJs accompanied by enhanced outward movement of the jet boundary in the orthogonal direction. Predicted mean axial velocity contours on the $\mathrm{x}-\mathrm{z}(\mathrm{y}=0)$ and $x-y(z=0)$ planes are shown in Fig. 11. The jet cross-section no longer remains axisymmetric; the contour shape changes observed due to steady CJ injection are very clear in these figures. A bifurcation of the primary jet can also be identified from the two peaks observable in the mean velocity contours in the $\mathrm{z}=0$ plane, implying that the peak velocity has moved away from the original jet centreline; this was also observed in the experimental study [2] see below. One implication of this is that the furthest downstream penetration of the nozzle exit peak velocity can no longer be judged by examining centreline conditions alone. However, for simplicity, the centreline definition of potential core length will still be retained in comparing CFD and measurements. The shape of the potential core is also quite different from the classical clean jet shape seen in Fig. 8. A conical-like shape is still present in the $y=0$ plane, but with the edges more curved and converging radially inwards initially much more rapidly; on the $\mathrm{z}=0$ plane the competing effects of shear-driven mixing (moving the peak velocity contour radially inward) but CJinduced secondary velocity (moving fluid outwards) effectively cancel out and the peak contour is almost horizontal except for the bifurcating portion.
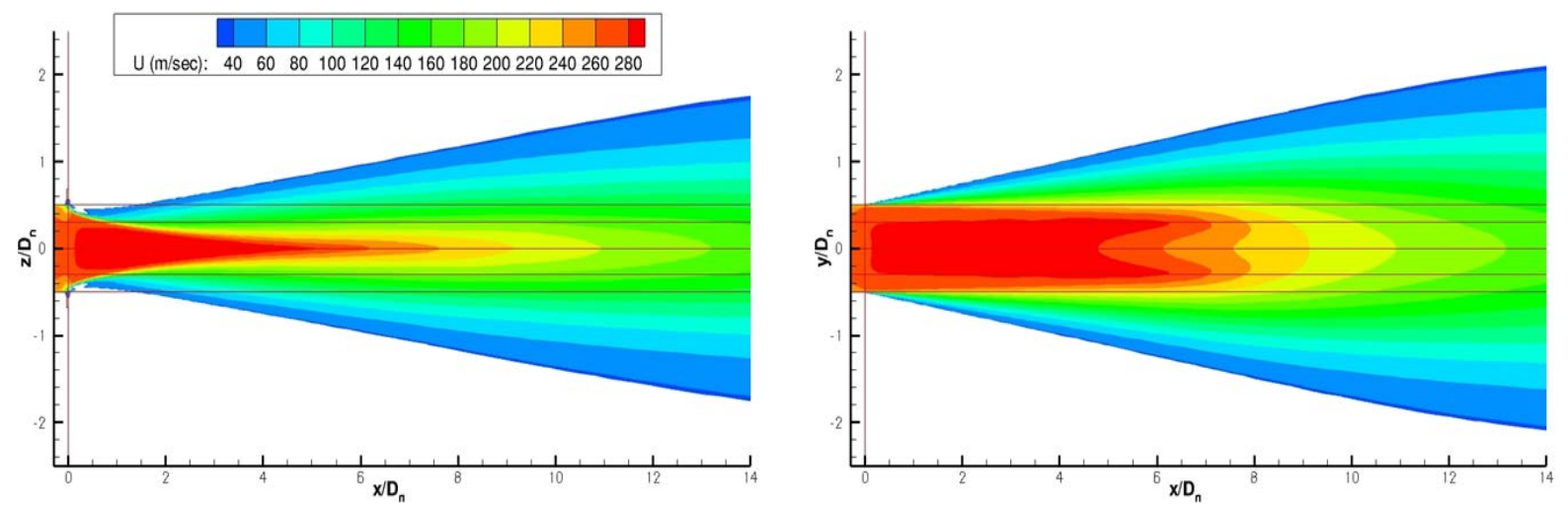

Fig. 11 Predicted axial velocity contours on $y=0$ (left) and $z=0$ (right) plane, steady CJs 
The fluid mechanics that brings these changes about is best visualised by examination of the cross-stream (V and W) secondary flows induced by the CJs. These are illustrated in Fig. 12 at several downstream axial locations via the
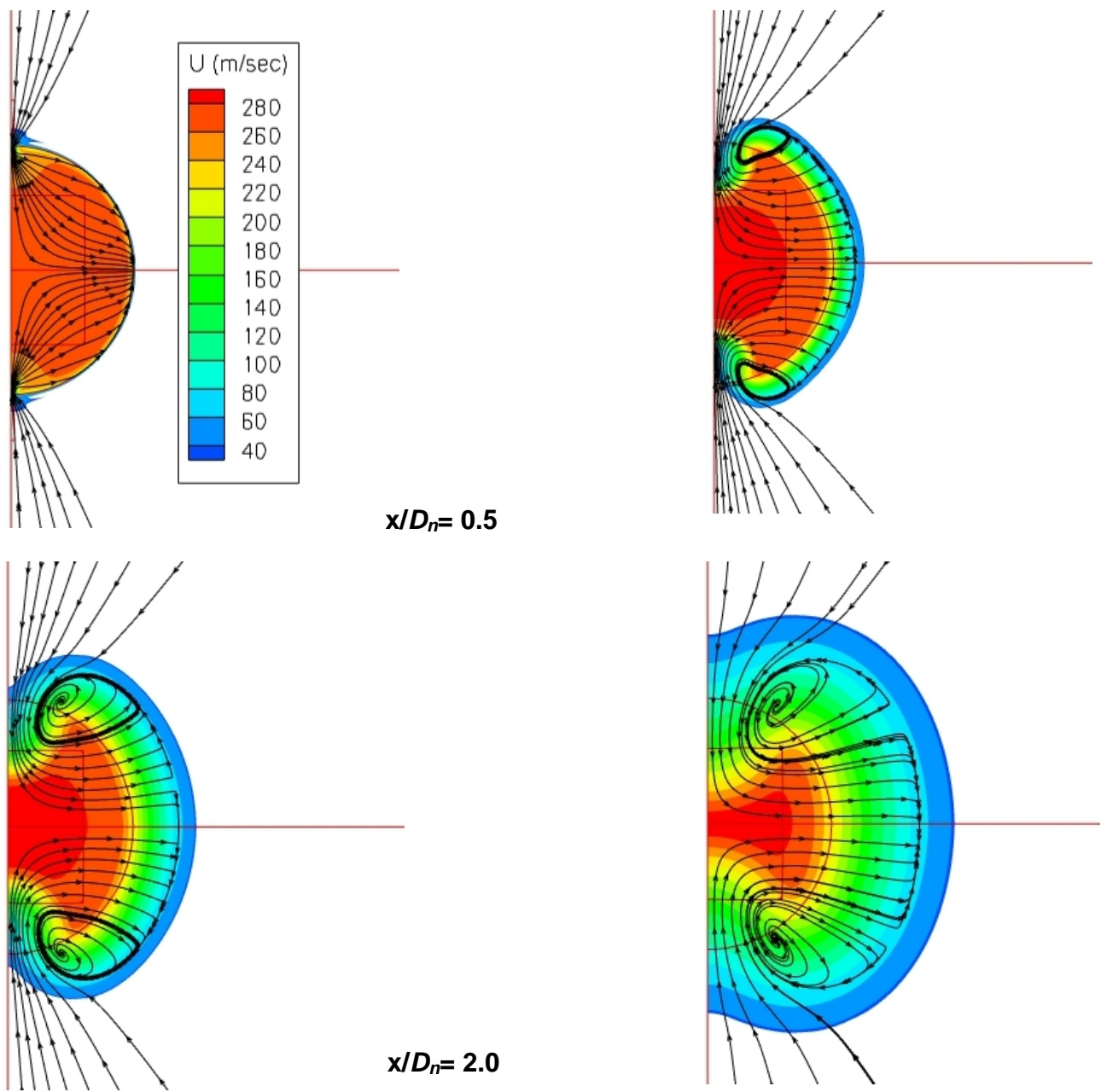

$x \mid D_{n}=1.0$
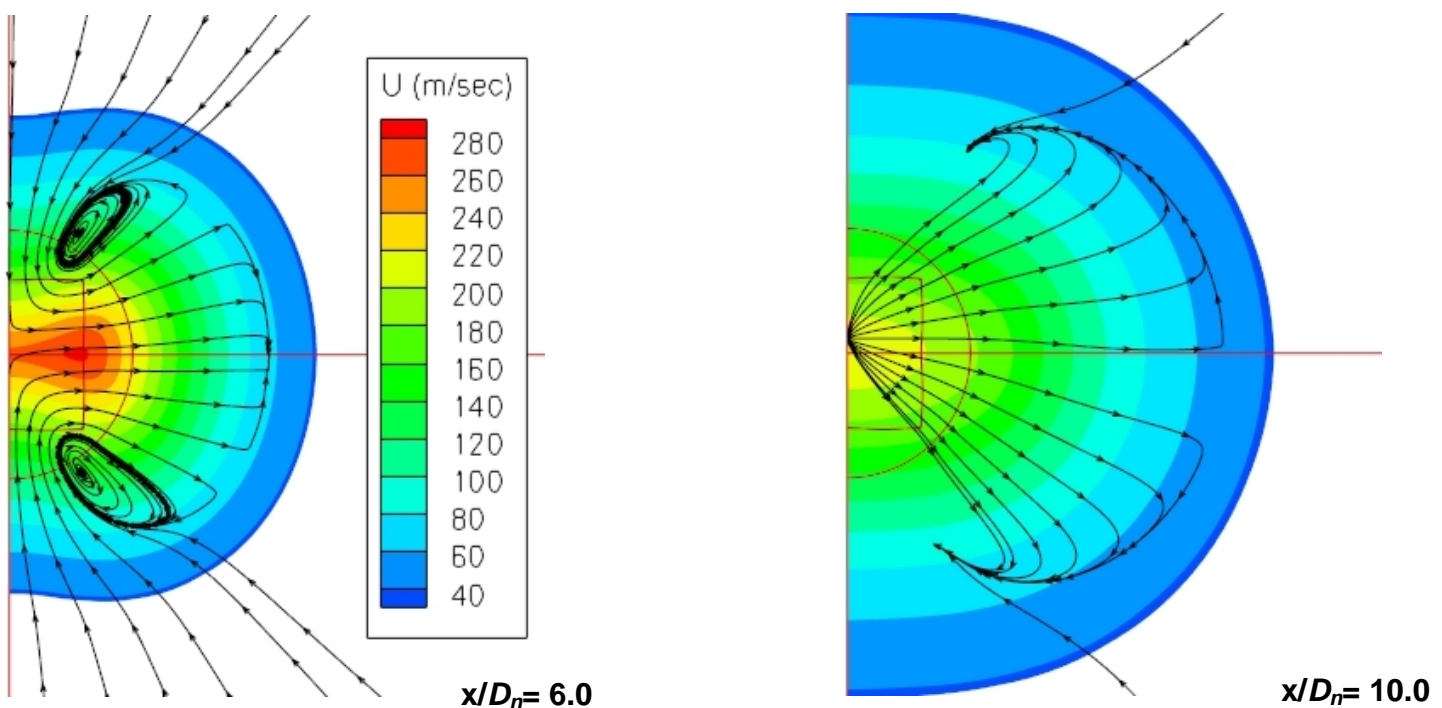

Fig. 12 Secondary velocity streamtraces and axial velocity contours at several axial stations, steady CJs 
superposition of axial velocity contours and streamtraces using just the in-plane secondary velocity components. These plots reveal a large radially inward flow is induced immediately by the $\mathrm{CJ}$ injection, creating a significant localised entrainment flow outside of the jet; continuity enforces a similar strong but radially more distributed outward flow in the orthogonal direction (y). These flows are what cause the contraction/expansion along/orthogonal to the CJ injection line (z direction). No sign of a streamwise vortex is seen at $\mathrm{x} / D_{n}=0.5$, but the development of the streamwise vortex pair created by each control jet is clearly identified in the predictions by $\mathrm{x} / D_{n}=1.0$ (only one vortex is shown in Fig. 12 due to symmetry). With increasing downstream distance the size and strength of the vortex grow, and the vortex centre moves in both azimuthal and radial directions, inducing increased jet spread. A non-circular jet cross-section is clearly identifiable; two factors are noteworthy: (i) larger spread in the y compared to the z-direction, and (ii) the largest z-dimension moves off the symmetry axis, with both jet edge and jet core acquiring a 'dumbbell' shape by $\mathrm{x} / D_{n}=4$; this is further evidence of bifurcation. From this distance downstream, the vortex begins to shrink and lose strength. At $\mathrm{x} / D_{n}=6$, although the vortex structure can still be seen, the concentration of closed streamtraces is limited to a small area and the secondary velocities are becoming smaller. From $\mathrm{x} / D_{n}=10$ and further downstream, the spread of the jet begins to resemble the clean jet characteristic, with jet fluid flowing equally outward in all directions. At this location the jet cross-section has an elliptical shape with the major(y)/minor (z) axis ratio being $~ 1.25$.

To determine the extent that the RANS k- $\varepsilon$ solution has captured these CJ-induced 3D processes, it is necessary to compare with experimental data. Predicted and measured non-dimensional centreline velocities for the case of steady CJs are shown in Fig. 13. The predicted potential core length is $4.7 D_{n}$, compared to the measured value of

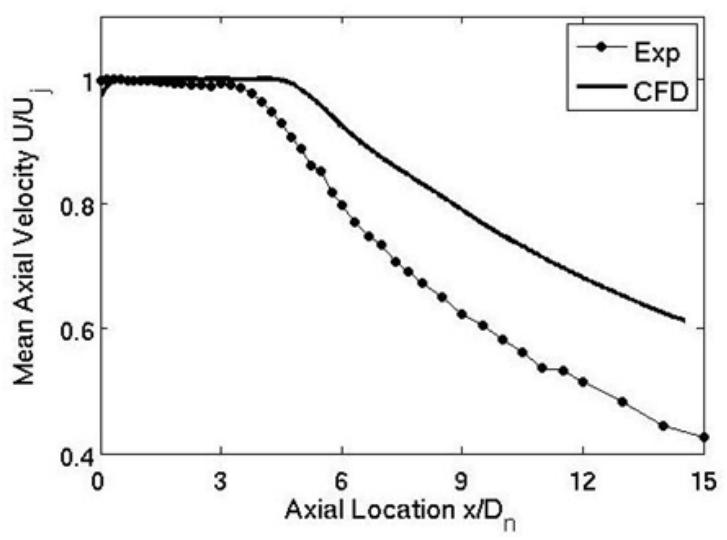

Fig. 13 Non-dimensional axial velocity on jet centreline, steady CJs - CFD and Expts.[2] 
$3.25 \mathrm{D}_{\mathrm{n}}$. If the baseline for CFD/Expt. comparison is taken as the (different) CFD/Expt. values for the baseline jet, this corresponds to a reduction of $43 \%$ (CFD) or $48 \%$ (Expt.). Thus, although the expected overprediction of absolute core length by an eddy viscosity RANS model is still present, the relative trend in potential core length reduction due to the action of steady CJs is predicted remarkably well.

To validate the RANS predictions of the 3D jet shape change, transverse (y and $\mathrm{z}$ ) axial velocity profiles are compared with measurements in Fig. 14. Very close to the nozzle exit plane $\left(\mathrm{x} / D_{n}=0.5\right)$, a stronger z-direction contraction of the jet is predicted compared to experiments; by $\mathrm{x} / D_{n}=3$ the difference between predictions and measurements has become very small; the different shear layer profiles in y- and z-directions are reproduced well. The too slow rate of mixing downstream of the potential core observed in the baseline jet shows up again for steady CJs in the overprediction of velocity on the symmetry plane. Evidence of jet bifurcation began to appear in the experiments at $\sim \mathrm{x} / D_{n}=4.0$, whereas bifurcation was observed in the predicted profile only at $\mathrm{x} / D_{n}=5$. The process of bifurcation continued in the predictions up to $\mathrm{x} / D_{n}=7$, after which vortex decay took over and the off-symmetry plane maximum moved back to the y-axis. The experimental and CFD profiles are almost parallel in the bifurcated
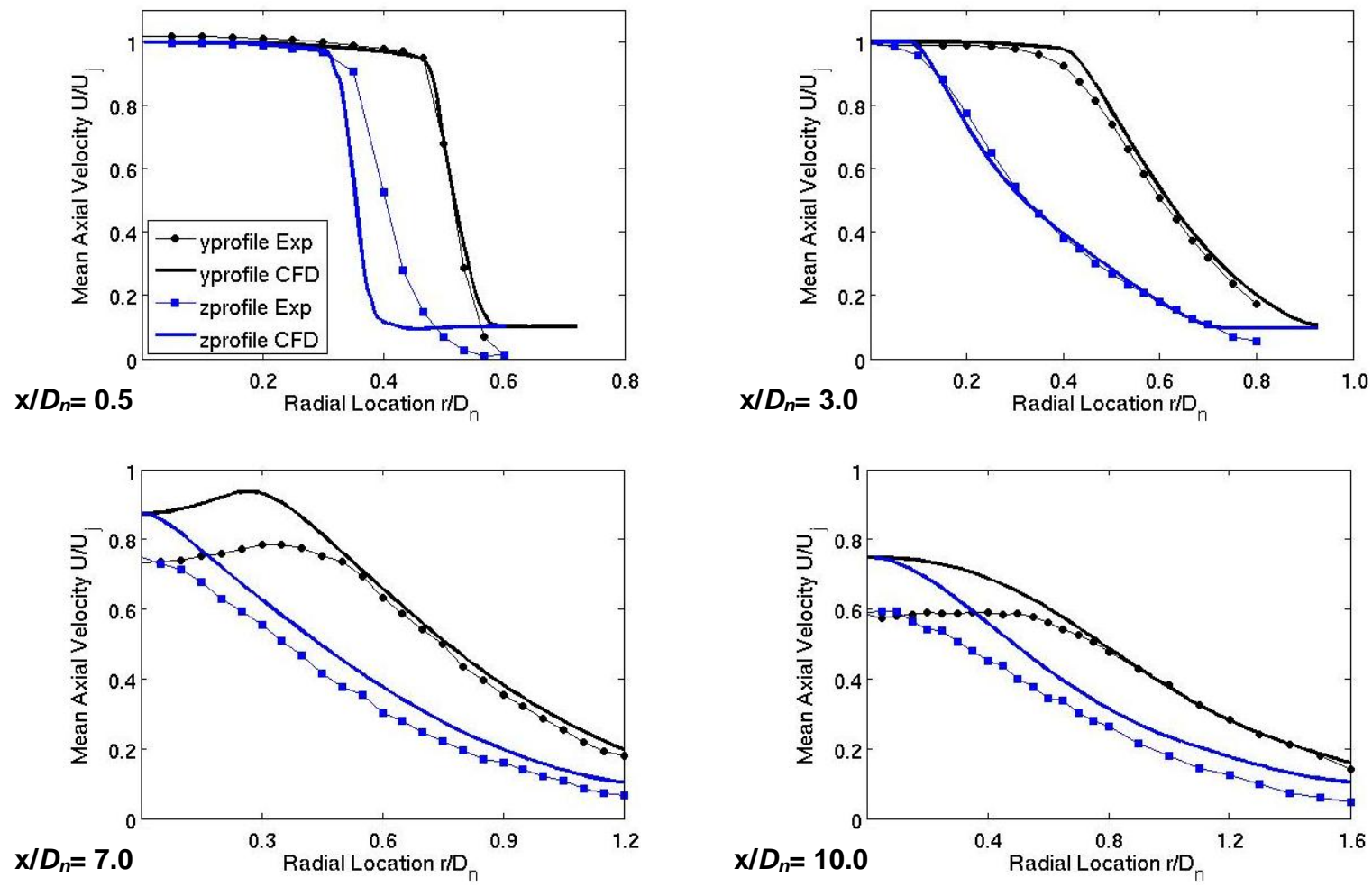

Fig. 14 Axial velocity profiles at different axial locations, steady CJs - CFD and Expts.[2] 
region. The bifurcation weakens as distance from nozzle exit plane increases, and no sign of a bifurcated jet is observed at $\mathrm{x}=10 \mathrm{D}_{\mathrm{n}}$ similar to the experimental findings. The profiles at $\mathrm{x} / D_{n}=10$ indicate that the contraction of the jet in the $\mathrm{z}$ direction in the far field is underpredicted, although the initial contraction was observed to be stronger. The conclusion is that both vortex formation and decay processes are predicted to be rather too rapid compared to experimental evidence.

\section{iii. Pulsed Control Jets - in phase (symmetric) and out of phase (antisymmetric) forcing}

Predictions were carried out for the conditions identified in the experiments ([2], [14]) as leading to optimum increase in mixing rate, i.e. a duty cycle $\alpha$ of $32 \%$ and at a pulsation frequency corresponding to a Strouhal number of $S t=0.22$. Analogous to the presentation of results for steady CJs, contour plots are first used to identify the flow structure, followed by quantitative comparison with measured long time-average measurements of the axial velocity.

\section{Symmetric Forcing}

The changes induced in the primary jet structure on the two orthogonal viewing planes used above $(\mathrm{y}=0$ and $\mathrm{z}=$ 0) when forced symmetrically (pulsed in phase) by two unsteady CJs are shown in Fig. 15. Two views are sufficient to characterise the flow pattern: (i) a single instantaneous snapshot at a time of $75 \%$ of the pulsation cycle $(\mathrm{t} / \mathrm{T}=3 / 4$, where $\mathrm{T}$ is the pulse period - other instants show similar features) (top), and (ii) the long time-averaged contour pattern (bottom). Examining first the long time-averaged results, it was found that the contraction of the jet edge in the $y=0$ plane with pulsed injection was weaker compared to steady injection at the same CJ supply pressure (compare Fig. 15 (bottom left) with Fig. 11 (left)). Similarly, in the $\mathrm{z}=0$ plane there is much less evidence of bifurcation (compare Fig. 15 (bottom right) with Fig.11 (right)). A weaker $y=0$ plane contraction with pulsed forcing compared to steady forcing was also observed in the experimental study [2]; however, measurements indicated no bifurcation in the $\mathrm{z}=0$ plane data but, as noted above, a slight bifurcation was still seen in the predictions. 

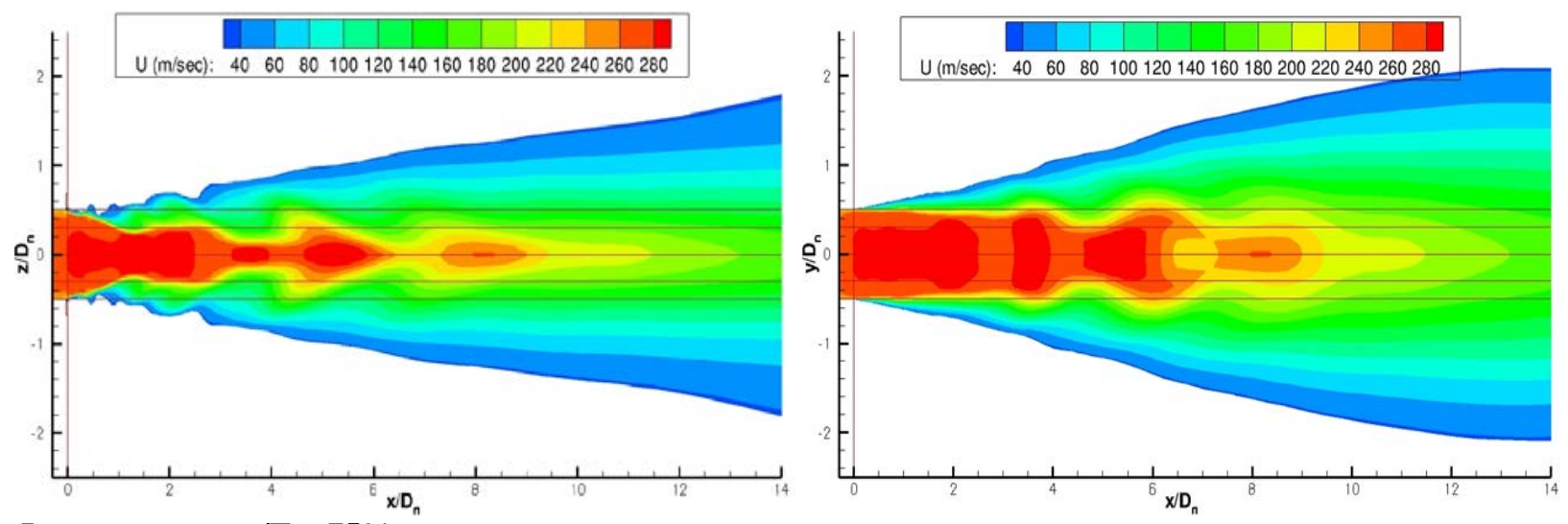

Instantaneous - $\mathbf{t} / \mathbf{T}=75 \%$
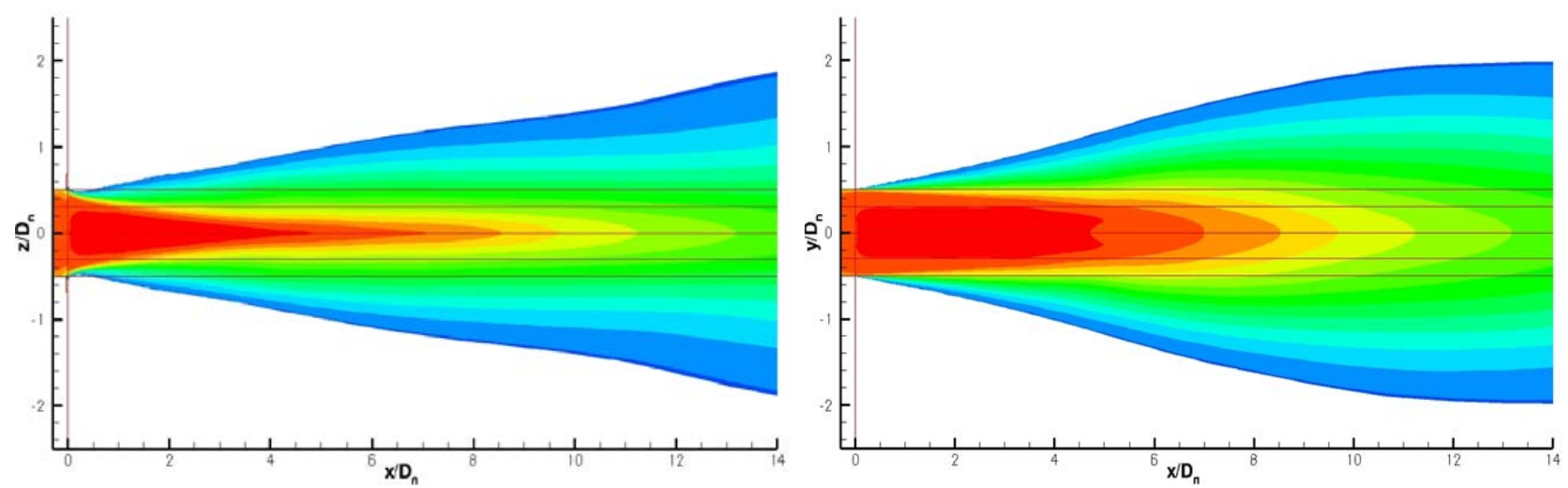

Time-averaged

Fig. 15 Axial velocity contours in $y=0$ plane (left) and $(\mathrm{z}=0$ plane (right) - pulsed CJs symmetric forcing

Some of the fluid mechanics behind the changes introduced by pulsed forcing is revealed in the instantaneous $(\mathrm{t} / \mathrm{T}=75 \%)$ images in Fig 15 (top). Oscillating the CJ supply pressure causes the injected radial momentum to be introduced in discrete 'slugs' or 'puffs' of fluid. After being turned into the streamwise direction by the crossflowing primary jet, these roll up into separate vortices, and the locations of these are easily distinguished by the wave-like contour shapes in the instantaneous snapshots. Note that the perturbations are of opposite sign in the two planes shown at any given axial location - when the contour moves radially inward on $y=0$, it moves outward on $\mathrm{z}$ $=0$; this is connected with the continuity-driven nature of the V velocity perturbation cause by the CJ-injected W velocity. The literature reviewed in the introduction is devoid of any explanation of the vortex structures induced by pulsed CJs (and how these change between different pulse modes). Identification of these structures has been carried out by examining one of the unsteady secondary velocity components. Since the CJs in the current study were introduced in the $\mathrm{z}$-direction, $\mathrm{W}$ velocity contours in the $\mathrm{y}=0$ plane are used to reveal the individual vortices and 
their movement. Instantaneous $\mathrm{W}$ contours are shown here for two different time instants $-\mathrm{t} / \mathrm{T}=25 \%$ (Fig. 16 (left)), and $\mathrm{t} / \mathrm{T}=75 \%$ (Fig. 16 (right)).

With in-phase forcing, the vortex structures are symmetric across the jet centreline and do not cross the centreline, due to the presence and motion of an equivalent and symmetrically opposed vortex structure. The vortex structures from both CJs move initially towards the centreline, but are then forced to move axially by the corresponding vortex from the opposite CJ. Notice that the vortex structures from successive CJ pulses are well separated. This spatial distance between the vortex structures is a function of both duty cycle and pulsation frequency. At a given frequency, a higher duty cycle results in a reduction of spatial separation and vortices then have less time to act independently, which makes a pulsed CJ to behave as a quasi-steady CJ. Similarly, at a given duty cycle, a higher frequency will shrink the time between successive pulses; hence spatial separation reduces. This is an equivalent aspect of pulsed CJs which determines the optimum number of tabs azimuthally around the primary jet perimeter. A small enough tab number/large enough vortex separation should be used to allow the vortices created to have maximum time to enhance mixing before they interact with each other. In Fig. 16 (left), four vortex structures per CJ can be seen. The first is just starting to form, the second is part way through the 'growth' phase, increasing in both size and strength, the third is at the end of the growth phase, and the fourth, some way downstream, has undergone significant decay. Fig. 16 (right) shows the vortex structures at 50\% of a period later (at $\mathrm{t} / \mathrm{T}=75 \%$, the pulsation is OFF). All structures seen in Fig. 16 (left) have been convected downstream by the primary jet velocity, except for the fourth vortex in Fig. 16(left), which has decayed completely. A small new vortex at the CJ injection location may just be seen.
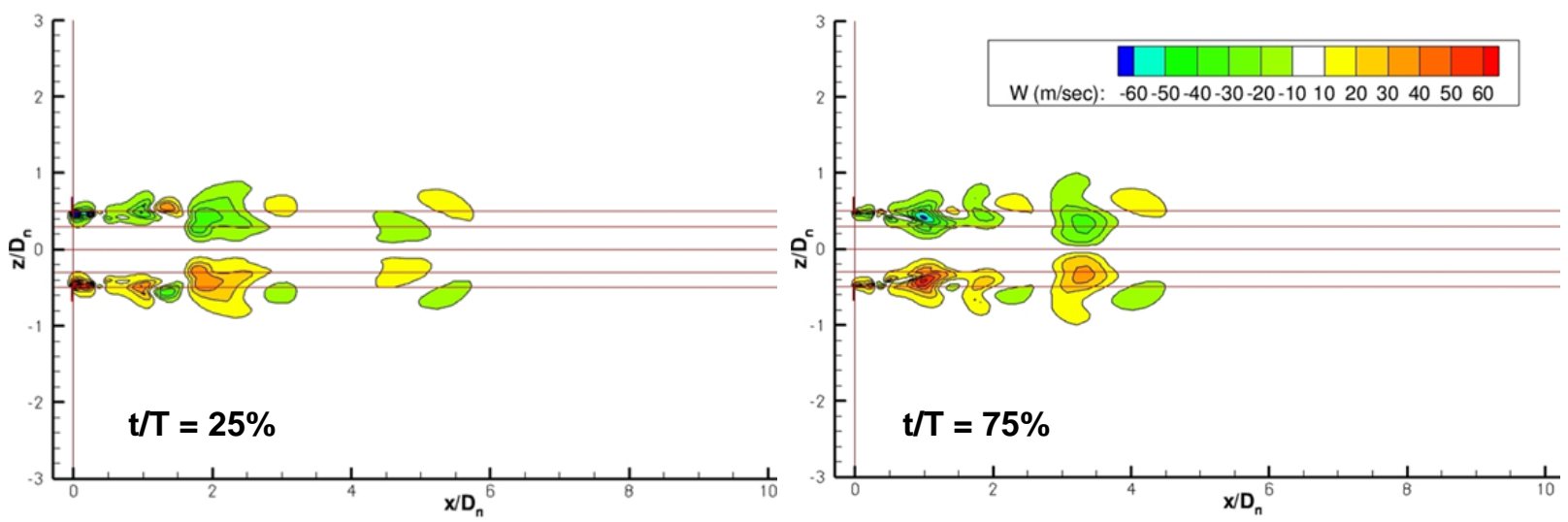

Fig. 16 Instantaneous radial velocity contours on $\mathbf{y}=0$ plane, pulsed CJs, symmetric forcing 
A quantification of the extent to which the pulsed CJ URANS CFD predictions have captured the unsteady vortex-induced mixing of axial momentum accurately is again made by comparing predictions with measured data. Starting as usual with potential core length, and using as indicated above a calculation based on the reduction relative to the equivalent (CFD or experiment) clean jet value, CFD predicted a $45 \%$ centreline reduction in core length $\left(4.5 D_{n}\right)$; experiments indicated a $40 \%$ reduction $\left(3.75 D_{n}\right)$. Once again, as for steady CJs, the trend of potential core length reduction was reproduced quantitatively well.

The long time-averaged axial velocity profiles on the two viewing planes are presented in Fig. 17 for 4 axial stations. The overall impression is that the change from steady to pulsed jets has not significantly altered the level of
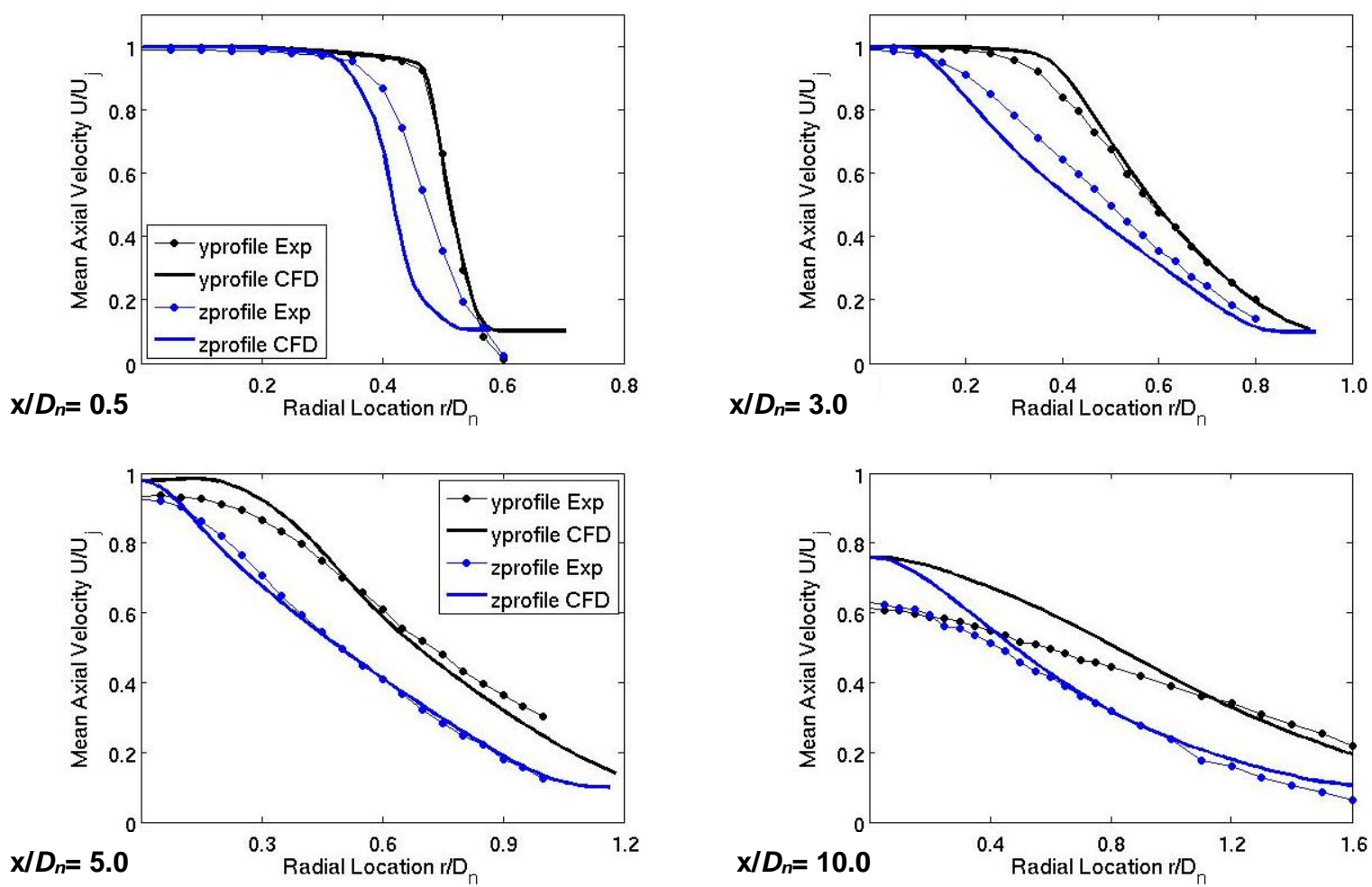

Fig. 17 Mean axial velocity profiles at different axial locations, pulsed CJs, symmetric forcing

agreement between CFD and measurements. Similar to the steady case, the initial contraction along the $\mathrm{z}$ direction is predicted to be more aggressive than measured. The bifurcation predicted in the pulsed case is just visible in Fig. 17 at $\mathrm{x} / \mathrm{D}_{\mathrm{n}}=5$, but it is clear the effect is much weaker than the steady case, and totally absent in the pulsed experiments. Further downstream, the usual divergence between predictions and data in the centreline region appears, but does 
not seem any worse than the clean or steady cases. The agreement away from the centreline is good, with the different locations of the shear layers in the two planes well predicted, as well as the location of the jet edge; this perhaps indicates that it is more the inner than the outer edge of the jet/ambient shear layer that is not well predicted by the turbulence model.

\section{Antisymmetric Forcing}

For the antisymmetric pulsed operating condition, the CJs operate with a phase difference of $180^{\circ}$. A flapping of the jet is induced, clearly illustrating the 'sinuous' description of this mode. This is identifiable in the instantaneous $\mathrm{y}=0$ plane snapshot at $\mathrm{t} / \mathrm{T}=75 \%$ in Fig. 18 (top left). Strong jet flapping is promoted by the vortex trajectories induced by an antisymmetric mode of pulsation; individual vortices are able to penetrate further into the jet due to the absence of a symmetrically opposite vortex structure. This causes even stronger bifurcation in the orthogonal plane than in the steady CJ case, as can be seen in both the instantaneous and long time-averaged $\mathrm{z}=0$ plane axial velocity contours (Fig. 18 (right)). The flapping action results in a significant reduction of the potential core length (compare Figs. 11, 15 and 18). A bifurcation of the primary jet was also noted in the experimental investigation [14], although the strength of bifurcation is again overpredicted as it was in the steady case.

To provide further insight into the vortex flow physics, the $\mathrm{W}$ velocity contours on the two usual viewing planes for the antisymmetric mode are shown in Fig. 19. There is again a time difference of T/2 between the two plots, thus, unlike in the symmetric forcing case, the velocity contours in the two plots now appear as mirror images of each other. Close observation and comparison with symmetric forcing shows that the initial movement of the vortex structures for both modes is identical, with the vortex structures moving towards the centreline of the jet and slightly downstream. A comparison of Figs. 16 and 19 at $\mathrm{t} / \mathrm{T}=25 \%$ shows that the vortex structures from the upper control jet are identical at $\mathrm{x} / D_{n}=2$, but there is a marked difference in the lower vortex structures at the same axial location. Contrary to the symmetric case, where neither vortex was able to reach the centreline due to the presence 

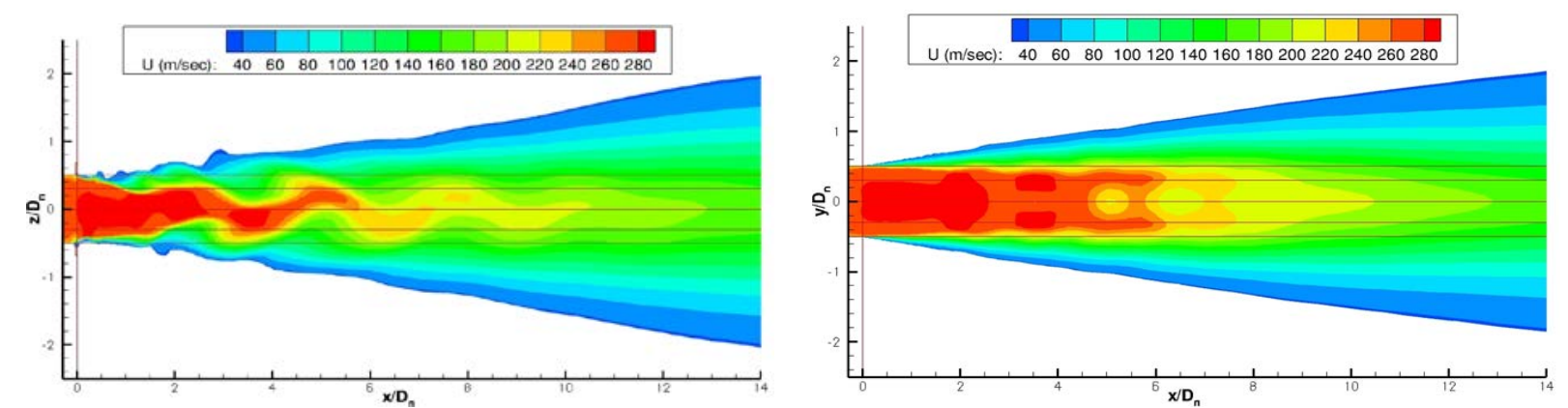

Instantaneous $-\mathbf{t} / \mathbf{T}=75 \%$
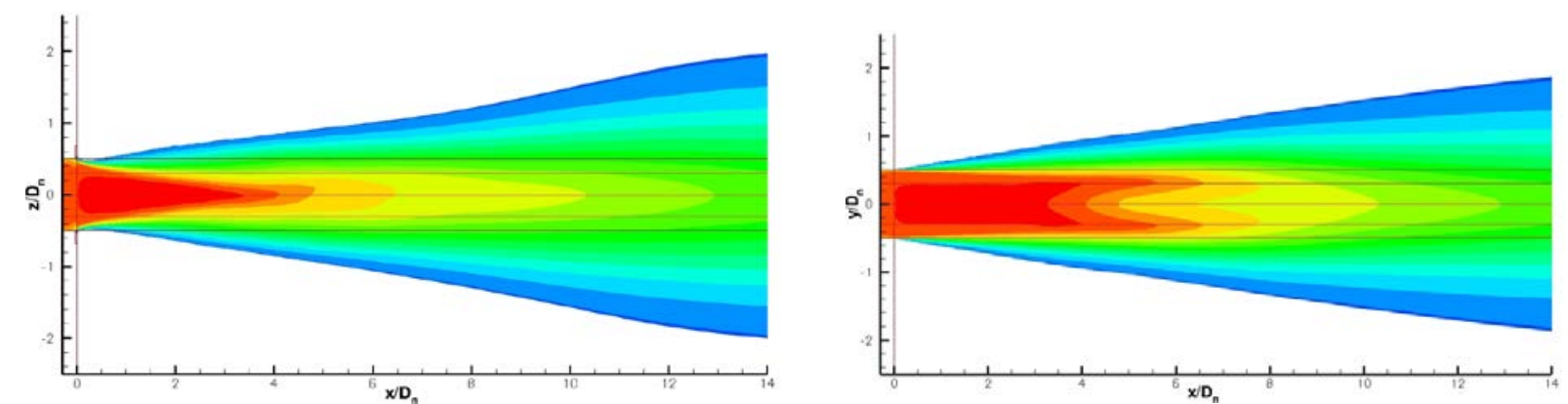

Time-averaged

Fig.18 Mean axial velocity contours on $y=0$ plane, pulsed CJs, antisymmetric forcing
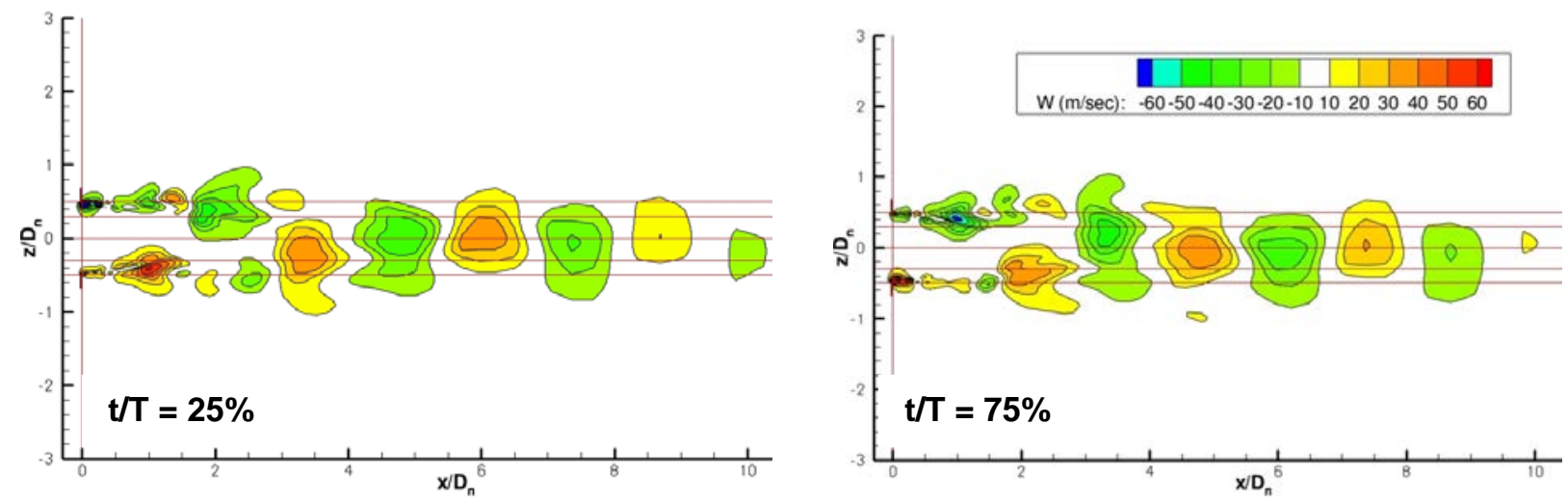

Fig. 19 Instantaneous radial velocity contours on $\mathbf{y}=\mathbf{0}$ plane, pulsed CJs, antisymmetric forcing

of a similar opposing vortex, the upper vortex in the antisymmetric case is able to reach and start to cross the nozzle centreline due to the weaker opposing vortex. This differing development of upper and lower vortices continues downstream, with alternate vortices extending across to the opposite nozzle lipline, thus undulating the core of the jet plume in the lateral direction (flapping), and reducing the centreline potential core length. Further, and more significantly, in the antisymmetric mode, the strength of the vortex structures is not reduced with downstream 
movement and vortices are predicted to be present as far as $\mathrm{x}=10 D_{n}$. A key point to observe in Figs. 16 and 18 is the difference of the vortex radial locations between the two modes. In the symmetric mode, the vortices stay within the shear layer; due to the strong velocity gradient in the shear layer, the effects of the vortices then decay much more rapidly in the symmetric mode. In the antisymmetric mode, the vortices are able to penetrate right across the jet core causing much larger perturbation in the core/shear layer interface. This seems to be a more effective mechanism for enhanced mixing (i.e. increasing the interfacial area across which mixing takes place), rather than the symmetric mechanism which is more akin to enhancing mixing via increased turbulence in the shear layer itself. In the experimental investigation, a comparison of the two azimuthal modes showed the antisymmetric mode to be more effective at enhancing jet spread ([2], [14]). The radial positioning of the vortices in the present numerical predictions has provided an explanation of this behaviour.

The potential core length predicted by URANS CFD for antisymmetric forcing was $3 D_{n}$ and matched the measured value exactly. In relative terms using the clean jet prediction/measurement as baseline, this implied pulsed antisymmetric forcing produced a 63\% reduction (CFD) and 51\% reduction (Expts.). The mean axial velocity profile development for antisymmetric forcing is presented in Fig. 20. The strength of bifurcation was overpredicted beyond potential core end. The areas of agreement/disagreement are once more on the whole similar to symmetric pulsed and steady forcing.

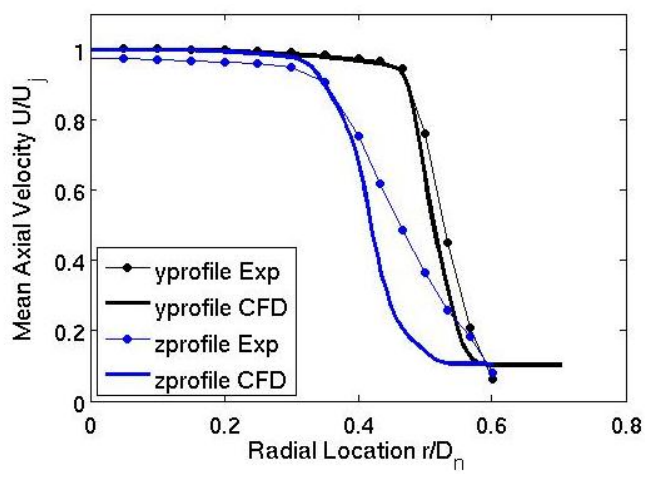

a) $\mathrm{x} / \mathrm{D}_{\mathrm{n}}=0.5$

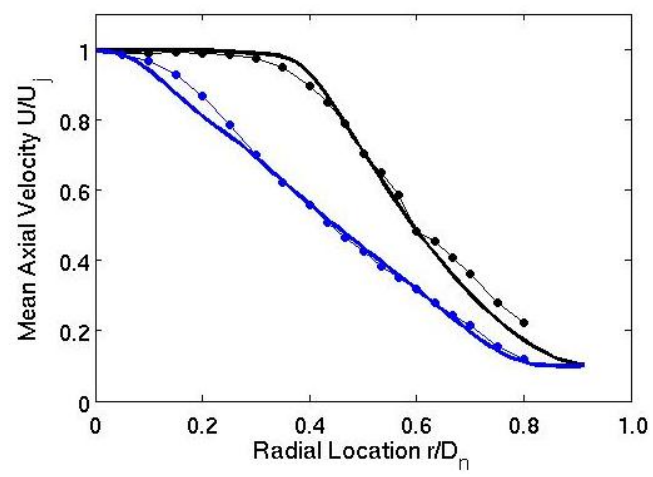

b) $\mathrm{x} / \mathrm{D}_{\mathrm{n}}=3$ 


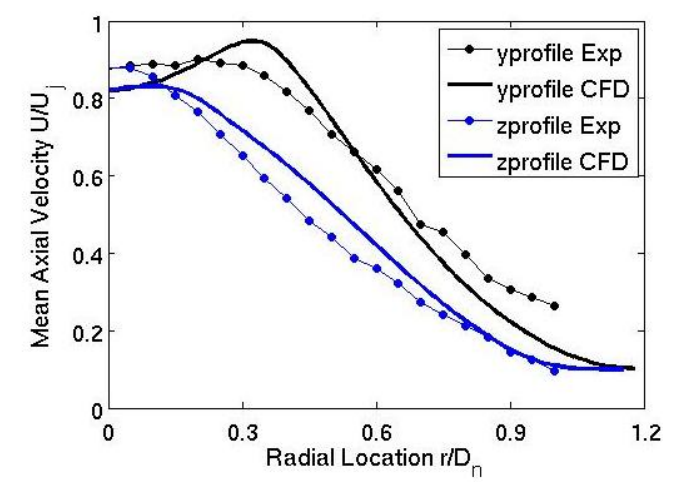

c) $x / D_{n}=5$

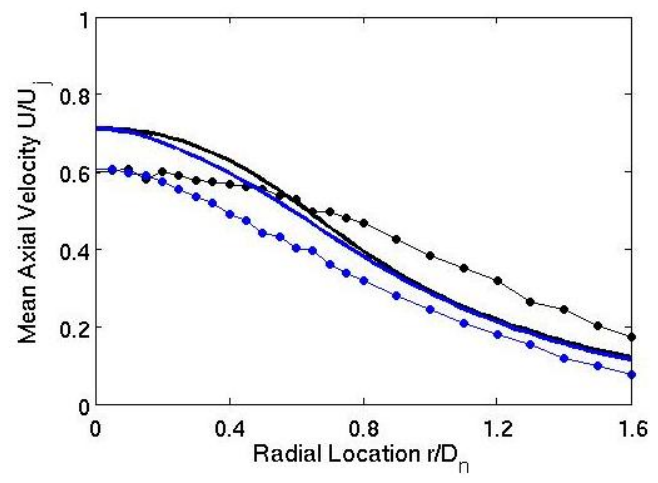

d) $\mathrm{x} / \mathrm{D}_{\mathrm{n}}=10$

Fig. 20 Mean axial velocity profiles at different axial locations, pulsed CJs, antisymmetric forcing.

\section{Conclusions}

The work presented here has focused on developing a better understanding of the ability of URANS-based CFD to capture, sufficiently accurately for early design studies, the physical mechanisms responsible for mixing enhancement caused by steady and pulsed CJs on high Re, high subsonic Mach number jets. URANS predictions were performed using a standard k- $\varepsilon$ turbulence model. A Mach 0.9 jet from a convergent nozzle was studied under 'clean' conditions (no control), with 2 steady CJs, and 2 pulsed CJs in symmetric/antisymmetric modes. URANS CFD captured the changes brought about by the CJs well. Although absolute lengths of the potential core for the various configurations differed from measurements, the relative effect of CJs was predicted well. For steady CJs, a $43 \%$ reduction compared to the clean case was predicted against an experimentally determined reduction of $48 \%$. Similarly, for pulsed jet forcing, reduction in core length was predicted of $45 \%$ (symmetrical) and $63 \%$ (antisymmetrical), while experiments indicated $40 \%$ and $51 \%$ reduction. Variations in the cross-sectional profile shape were also captured satisfactorily. Initial contraction along the CJ introduction plane was slightly overpredicted for all cases, but beyond $\mathrm{x} / D_{n}=7$ the contraction was underpredicted. This showed that vortex formation and decay processes were both predicted to be too rapid. URANS was able to capture the bifurcation noted in the experiments. This study has indicated that it is more the inner than the outer edge of the jet/ambient shear layer that is not well predicted by URANS using standard k-e. Analysis of predictions was used to illustrate how the presence of a CJinduced streamwise vortex pair was responsible for enhanced mixing. Pulsed CJs in symmetric/antisymmetric modes influenced the movement and relative strength of the induced vortices. In the symmetric mode, the vortices stayed within the shear layer and decayed rapidly. However, in antisymmetric mode, the vortices were able to 
penetrate across the jet core causing much larger perturbation in the core/shear layer interface, increasing the interfacial area and surviving longer. This explained the superiority of antisymmetric over symmetric mode in enhancing the jet spreading rate, as noted in the experiments. Further work should explore the possibility that a noneddy viscosity based turbulence model (i.e. a Reynolds Stress Transport (RST) model) could improve the absolute level of accuracy of URANS for this problem. Carpy and Manceau [31] have shown that this can improve the accuracy of isolated pulsed jet prediction, but it is not clear whether this would remove the absolute error in potential core length prediction; this is worthy of exploration.

\section{References}

[1] Afsar, M. Z., Hynes, T. P., Dowling, A. P., McMullan, W. A., Pokora, C., Page, G. J., and McGuirk, J. J., "Jet Noise: Acoustic Analogy informed by Large Eddy Simulation”, AIAA Jnl., Vol. 48, 2010, 1312-1325.

[2] Kamran, M. A., and McGuirk, J., "Subsonic Jet Mixing via Active Control Using Steady and Pulsed Control Jets ", AIAA Journal, Vol. 49, 2011, 712-724.

[3] Behrouzi, P. and McGuirk, J., "Jet Mixing Enhancement Using Fluid Tabs", AIAA 2004-2401, Proc. of $2^{\text {nd }}$ AIAA Flow Control Conference, Portland, Oregon, USA, 2004.

[4] Davis, M.R., "Variable Control of Jet Decay", AIAA Journal, Vol. 20, 1982, 606-609.

[5] Lardeau, S., Collin, E., Lamballais, E., Delville, J., Barre, S., Bonnet, J.P., "Analysis of A Jet-Mixing Layer Interaction", International Journal of Heat and Fluid Flow, Vol. 24, 2003, 520-528.

[6] Tamburello, D. A., and Amitay, M., "Manipulation of an Axisymmetric Jet using Continuous Control Jets”, Journal of Turbulence, Vol. 7, 2006, 1-24.

[7] Tamburello, D. A. and Amitay, M., “Interaction of a Free Jet with a Perpendicular Control Jet”, Jnl. of Turbulence, Vol. 8, 2007, 1-27.

[8] Crow, S.C. and Champagne, F.H., "Orderly Structure in Jet Turbulence", Jnl. of Fluid Mech., Vol. 48, 1971, $547-591$.

[9] Raman, G., and Cornelius, D., "Jet Mixing Control Using Excitation From Miniature Oscillating Jets", AIAA Journal., Vol. 33, 1995, 365-368.

[10] Parekh, D.E., Kibens, V., Glezer, A., Wiltse, J.M., Smith, D. M., "Innovative Jet Flow Control: Mixing Enhancement Experiments", AIAA Paper 96-0308, 1996.

[11] Ibrahim, M.K., Kunimura, R., and Nakamura, Y., "Mixing Enhancement Of Compressible Jets By Using Unsteady Microjets As Actuators", AIAA Journal, Vol. 40, 2002, 681-688.

[12] Behrouzi, P. and McGuirk, J., "Flow Control Of Jet Mixing Using A Pulsed Fluid Tab Nozzle", AIAA 2006-3509, $3^{\text {rd }}$ AIAA Flow Control Conference, San Francisco, California, 2006. 
[13] Behrouzi, P., Feng, T., McGuirk, J.J., "Active Control of Jet Mixing Using Steady and Pulsed Fluid Tabs", Proc. IMechE Part I: Jnl. of Systems and Control Engineering, Vol. 222, 2008, 381-392.

[14] Kamran, M. A., “Manipulation of High Mach Number Shear Layers Using Control Jets”, PhD Thesis, Loughborough University, UK, 2009.

[15] Ritchie, B. D., Majumdar, D. R., Seitzman, J. M., “Mixing in Co-axial Jets using Synthetic Jet Actuators”, AIAA2000-0404.

[16] Tamburello, D. A., and Amitay, M., “Active Control of a Free Jet using a Synthetic Jet”, International Journal of Heat and Fluid Flow, Vol. 29, 2008, 967-984.

[17] Freund, J.B., and Moin, P., "Jet Mixing Enhancement by High-Amplitude Fluidic Excitation", AIAA Journal, Vol. 38, 2000, 1863-1870.

[18] Hilgers, A. and Boersma, B. J., “Optimisation of Turbulent Jet Mixing”, Fluid Dynamics Research, Vol. 29, 2001, 345-368.

[19] Lardeau, S., Lambellais, and Bonnet, Jean-Paul, “Direct Numerical Simulation of a Jet Controlled by Fluid Injection”, Journal of Turbulence, Vol. 3, 2002, 1-25.

[20] Smith, T.D., Cain, A.B., and Chenault, C.F., "Numerical Simulation of Enhanced Mixing in Jet Plumes using Pulsed Blowing", Journal of Aircraft, Vol. 38, 2001, 458-463.

[21] Chauvet, N., Deck, S., and Jacquin, L., "Numerical Study Of Mixing Enhancement In A Supersonic Round Jet", AIAA Journal, Vol. 45, 2007, 1675-1687.

[22] Chauvet, N., Deck, S., and Jacquin, L., "Zonal Detached Eddy Simulation of a Controlled Propulsive Jet", AIAA Journal, Vol. 45, 2007, 2458-2473.

[23] Georgiadis, N. J., Yoder, D. A., Engblom, W. A., “Evaluation of Modified Two-Equation Turbulence Models for Jet Flow Predictions”, AIAA Journal, Vol. 44, 2006, 3107-3114.

[24] Georgiadis, N. J. and DeBonis, J. R., “Navier-Stokes analysis methods for turbulent jet flows with application to aircraft exhaust nozzles”, Progress in Aerospace Sciences, Vol. 42, 2006, 377-418.

[25] Thies, A. T. and Tam, C. K. W., “Computation of Turbulent Axisymmetric and non-axisymmetric Jet Flows using the k- $\varepsilon$ Model”, AIAA Journal, Vol. 34, 1996, 309-316.

[26] Andersson, B., Erikson, L-R, Davidson, L., “Large Eddy Simulation of Subsonic Turbulent Jets and Their Radiated Sound”, AIAA Journal, Vol. 43, 2005, 1899-1912.

[27] Wang, P. C. and McGuirk, J. J., “Large Eddy Simulation of Supersonic Jet Plumes from Rectangular Con-Di Nozzles”, Int. Journal of Heat and Fluid Flow, Vol. 43, 2013, 62-73.

[28] Launder, B.E. and Spalding, D.B., "The Numerical Computation of Turbulent Flows," Computational Methods in Applied Mechanical Engineering, Vol. 3, 1974, 269-289.

[29] Page, G.J., Zhao, H., and McGuirk, J.J., "A Parallel Multi-Block Reynolds-Averaged Navier-Stokes Method for Propulsion Installation Applications," Proc. of 12th International Symposium on Air Breathing Engines, Vol. 1, 1991, 864-875. 
[30] Birkby, P. and Page, G. J., "Numerical predictions of turbulent under-expanded sonic jets using a pressure-based methodology." Proc. of Inst. of Mechanical Engineers, Part G: Journal of Aerospace Engineering Vol. 215, 2001, $165-173$.

[31] Carpy, S. and Manceau, R., “Turbulence modelling of statistically periodic flows; Synthetic jet into quiescent air”, Int. Journal of Heat and Fluid Flow, Vol. 27, 2006, 756-767. 